Research Article

\title{
Topologies Generated by Two Ideals and the Corresponding j-Approximations Spaces with Applications
}

\author{
Mona Hosny (iD) 1,2 \\ ${ }^{1}$ Mathematics Department, Faculty of Science, King Khalid University, Abha, Saudi Arabia \\ ${ }^{2}$ Mathematics Department, Faculty of Education, Ain Shams University, Cairo, Egypt \\ Correspondence should be addressed to Mona Hosny; monahosny@edu.asu.edu.eg
}

Received 15 July 2021; Revised 18 August 2021; Accepted 22 September 2021; Published 23 October 2021

Academic Editor: Ljubisa Kocinac

Copyright $@ 2021$ Mona Hosny. This is an open access article distributed under the Creative Commons Attribution License, which permits unrestricted use, distribution, and reproduction in any medium, provided the original work is properly cited.

Ideal is a fundamental concept in topological spaces and plays an important role in the study of topological problems. This motivated us to use two ideals to generate different topologies to take the advantage of the two ideals at the same time. Two ideals represent two opinions instead of one opinion which is very useful for using the insights of two groups of experts to study the problem and elicit decisions based on their common vision. Topology is a rich source for constructs that is helpful to enrich the original model of approximations spaces. Rough set theory has inbuilt topological concepts. Hence, the main purpose of this paper is to point out that the concept of rough sets has a purely topological aspects nature. To do so, new approximations spaces are introduced and defined based on the topologies generated by two ideals. The results in this paper show that the topological concepts can be a powerful method to study rough set models. The basic properties of these approximations are studied and compared to the previous ones and shown to be more general. The importance of the current paper is not only introducing a new kind of rough set based on bi-ideals, increasing the accuracy measure, and reducing the boundary region of the sets which is the main aim of rough set but also introducing a chemical application to explain the concepts.

\section{Introduction}

It is well known that Pawlak's rough set theory $[1,2]$ is constructed on the basis of an equivalence relation or a partition. An equivalence relation or a partition plays an important role in Pawlak's rough set; however, it seems to be restrictive for many applications. Many generalizations to Pawlak space in order to change the constraints of the equivalence relation have been proposed [3-8]. Topology is a useful theoretic framework for the study of this theory as the topological notions are closely related to the notions and results of this theory. Lower and upper approximations operators are the central concepts in this theory and have the properties of the closure and interior operators. So, approximations operators in the rough sets theory are topological operators. Hence, the conjoint investigation of the rough set theory and topology becomes essential. Therefore, there are several generalizations of this theory, and each generalization employs topological concepts. The concept of the topological rough set by Wiweger [9] in 1989 is one of the most important topological generalizations of rough sets. The significant conjoint studies of the rough set theory and topology have been done by many researchers [9-23]. Ideal in topological spaces has been considered since 1930. This concept has won its importance by Vaidyanathaswamy [24]. In 1990, Jankovic and Hamlett [25] further introduced the ideal topological spaces and their applications to various fields. After the advent of the concept of ideals, there have been many great attempts, so far, by topologists to apply the notion of ideals for manoeuvring investigations of different problems of topology. So, many researchers [26-33] were interested in applying the concept of the ideals in the rough set theory. Abd El-Monsef et al. [34] introduced mixed neighborhood systems to approximate the rough sets. After that, Abd El-Monsef et al. [35] applied the concept of " $j$-neighborhood space" (briefly, $j$-NS) to generalize the classical rough set theory by using different general topologies induced from binary relations. In 2020, Hosny [28] 
generated different topologies by using the notion of ideals. Additionally, she defined the approximations by using these topologies as a generalization of $[34,35]$.

This paper aims to study a certain extension problem via two ideals. Aware of the fact that ideals play an important role in the study of topological problems, this work is focused on generating different topologies by using two ideals instead of one. Moreover, the current study highlights the interdependencies of these topologies and the classical rough set theory. This paper comprises 6 sections and its sequence is as follows. Section 2 outlines some definitions and basic concepts of topology and the rough set theory. Different topologies are induced by two ideals in Section 3. The comparisons between these topologies are introduced. The present topologies are more general than the previous ones in $[28,35]$. It is shown that these topologies are finer than the previous ones. The current definitions coincide with Abd ElMonsef et al.'s [35] definitions if the two ideals are empty set and coincided with Hosny's [28] definitions if the two ideals are equal to each other. So, the previous definitions [28, 35] are a special case of the current definitions. Section 4 contains two methods to propose new approximation spaces by using two ideals. In the first method, the lower approximations are defined by the union of two lower approximations, whereas the upper approximations are defined by the intersection of two upper approximations. The second one is based on the generated topologies which are introduced in the previous section. Moreover, the main properties of these methods are presented. These methods are an extension of the previous approximations in $[28,35]$. The present methods reduced the boundary region and the current accuracy is greater than the previous ones. At the end of this section, the relationships among the current approximations are summarized in Table 1. It should be noted that even though Kandil et al. [31] presented two kinds of approximations via ideals in which one of them was based on one ideal and the other depended on two ideals, both of them depended on one neighborhood only. It is considered to be the main difference between these approximations and the current approximations which are based on open (closed) sets of topology (family of closed sets) generated by $j$-neighborhood and two ideals. Thus, there exist many differences between the main properties of the current approach and the previous one [31]. At the end of this work, an applied example is suggested in the chemistry field by using the current methods to illustrate the definitions in a friendly way. Section 6 compiles the conclusion of this work.

\section{Preliminaries}

Definition 1 (see [25]). A nonempty collection $\mathscr{I}$ of subsets of a set $U$ is called an ideal on $U$ if it satisfies the following conditions:

(1) $A \in \mathscr{I}$ and $B \in \mathscr{I} \Rightarrow A \cup B \in \mathscr{I}$

(2) $A \in \mathscr{I}$ and $B \subseteq A \Rightarrow B \in \mathscr{I}$
That is, $\mathscr{I}$ is closed under finite unions and subsets.

Definition 2 (see [31]). Let $\mathscr{I}_{1}, \mathscr{I}_{2}$ be two ideals on a nonempty set $U$. The smallest collection generating by $\mathscr{I}_{1}, \mathscr{I}_{2}$ is denoted by $\mathscr{I}_{1} \vee \mathscr{I}_{2}$ and defined as

$$
\mathscr{I}_{1} \vee \mathscr{I}_{2}=\left\{G \cup F: G \in \mathscr{I}_{1}, F \in \mathscr{I}_{2}\right\} \text {. }
$$

Proposition 1 (see [31]). If $\mathscr{I}_{1}, \mathscr{I}_{2}$ are two ideals on a nonempty set $U$ and $A, B$ are two subsets of $U$. Then, the collection $\mathscr{I}_{1} \vee \mathscr{I}_{2}$ satisfies the following conditions:

(1) $\mathscr{I}_{1} \vee \mathscr{I}_{2} \neq \phi$

(2) $A \in \mathscr{I}_{1} \vee \mathscr{I}_{2}, B \subseteq A \Rightarrow B \in \mathscr{I}_{1} \vee \mathscr{I}_{2}$

(3) $A, B \in \mathscr{I}_{1} \vee \mathscr{I}_{2} \Rightarrow A \cup B \in \mathscr{I}_{1} \vee \mathscr{I}_{2}$

It means that the collection $\mathscr{I}_{1} \vee \mathscr{I}_{2}$ is an ideal on $U$.

Definition 3 (see [35]). Let $R$ be an arbitrary binary relation on a nonempty finite set $U$. The $j$-neighborhood of $x \in U\left(N_{j}(x)\right), j \in\{r, l,\langle r\rangle,\langle l\rangle, i, u,\langle i\rangle,\langle u\rangle\}$ is defined as

(1) $r$-neighborhood: $N_{r}(x)=\{y \in U: x R y\}$

(2) l-neighborhood: $N_{l}(x)=\{y \in U: y R x\}$

(3) $\langle r\rangle$-neighborhood: $N_{\langle r\rangle}(x)=\cap_{x \in N_{r}(y)} N_{r}(y)$

(4) $\langle l\rangle$-neighborhood: $N_{\langle l\rangle}(x)=\cap_{x \in N_{l}(y)} N_{l}(y)$

(5) $i$-neighborhood: $N_{i}(x)=N_{r}(x) \cap N_{l}(x)$

(6) $u$-neighborhood: $N_{u}(x)=N_{r}(x) \cup N_{l}(x)$

(7) 〈i〉-neighborhood: $N_{\langle i\rangle}(x)=N_{\langle r\rangle}(x) \cap N_{\langle l\rangle}(x)$

(8) $\langle u\rangle$-neighborhood: $N_{\langle u\rangle}(x)=N_{\langle r\rangle}(x) \cap N_{\langle l\rangle}(x)$

Definition 4 (see [35]). Let $R$ be an arbitrary binary relation on a nonempty finite set $U$ and let $\xi_{j}: U \longrightarrow P(U)$ be a mapping which assigns for each $x$ in $U$ its $j$-neighborhood in $P(U)$. The triple $\left(U, R, \xi_{j}\right)$ is called a $j$-neighborhood space (briefly, $j-\mathrm{NS}$ ).

Theorem 1 (see [35]). Let $\left(U, R, \xi_{j}\right)$ be a $j-N S$, and $A \subseteq U$. Then, $\forall j \in\{r, l,\langle r\rangle,\langle l\rangle, i, u,\langle i\rangle,\langle u\rangle\}$, the collection $\tau_{j}=\left\{A \subseteq U: \forall p \in A, N_{j}(p) \subseteq A\right\}$ is a topology on $U$.

Definition 5 (see [35]). Let $\left(U, R, \xi_{j}\right)$ be a $j-N S$. A subset $A \subseteq U$ is called a $j$-open set if $A \in \tau_{j}$, and the complement of a $j$-open set is called a $j$-closed set. The family $\Gamma_{j}$ of all $j$-closed sets of a $j$-neighborhood space is defined by $\Gamma_{j}=\left\{F \subseteq U: F^{\prime} \in \tau_{j}\right\}$, where $F^{\prime}$ is the complement of $F$.

Definition 6 (see [35]). Let $\left(U, R, \xi_{j}\right)$ be a $j-\mathrm{NS}, A \subseteq U$, and $\forall j \in\{r, l,\langle r\rangle,\langle l\rangle, i, u,\langle i\rangle,\langle u\rangle\}$. The j-lower, $j$-upper approximations, $j$-boundary regions, and $j$-accuracy of $A$ are defined, respectively, as 


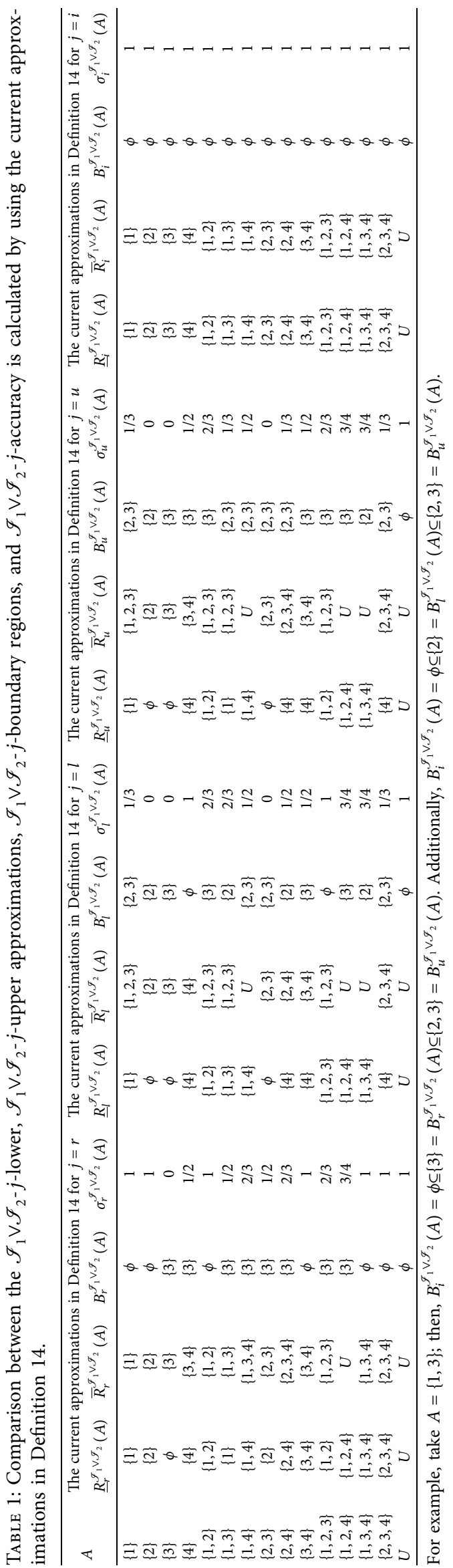




$$
\begin{aligned}
& \underline{\underline{R}}_{j}(A)=\cup\left\{G \in \tau_{j}: G \subseteq A\right\}=\operatorname{int}_{j}(A), \quad \text { where } \operatorname{int}_{j}(A) \text { represents } j \text { interior of } A, \\
& \bar{R}_{j}(A)=\cap\left\{H \in \Gamma_{j}: A \subseteq H\right\}=\mathrm{cl}_{j}(A), \text { where } \mathrm{cl}_{j}(A) \text { represents } j \text { closure of } A, \\
& B_{j}(A)=\bar{R}_{j}(A)-\underline{R}_{j}(A), \\
& \sigma_{j}(A)=\frac{\left|\underline{-}_{j}(A)\right|}{\left|\bar{R}_{j}(A)\right|}, \quad \text { where }\left|\bar{R}_{j}(A)\right| \neq 0 .
\end{aligned}
$$

Definition 7 (see [35]). Let $\left(U, R, \xi_{j}\right)$ be a $j-\mathrm{NS}$ and $\forall j \in\{r, l,\langle r\rangle,\langle l\rangle, i, u,\langle i\rangle,\langle u\rangle\}$. A subset $A \subseteq U$ is called a $j$-exact set if $\bar{R}_{j}(A)=\underline{R}_{j}(A)$. Otherwise, $A$ is called a j-rough set.

Theorem 2 (see [28]). Let $\left(U, R, \xi_{j}\right)$ be a $j-N S, A \subseteq U$, and $\mathscr{I}$ an ideal on $U$. Then, $\forall j \in\{r, l,\langle r\rangle,\langle l\rangle, i, u,\langle i\rangle,\langle u\rangle\}$, and the collection $\tau_{j}^{\mathscr{J}}=\left\{A \subseteq U: \forall p \in A, N_{j}(p) \cap A^{\prime} \in \mathscr{I}\right\}$ is a topology on $U$.

Theorem 3 (see [28]). Let $\left(U, R, \xi_{j}\right)$ be a $j-N S, A \subseteq U$, and $\mathscr{I}$ an ideal on $U$. Then, $\forall j \in\{r, l,\langle r\rangle,\langle l\rangle, i, u,\langle i\rangle,\langle u\rangle\}, \tau_{j} \subseteq \tau_{j}^{\mathscr{I}}$.
Definition 8 (see [28]). Let $\left(U, R, \xi_{j}\right)$ be a $j-\mathrm{NS}$ and $\mathscr{I}$ an ideal on $U$. A subset $A \subseteq U$ is called an $\mathscr{I}_{j}$-open set if $A \in \tau_{j}^{\mathscr{F}}$ and the complement of an $\mathscr{I}_{j}$-open set is called an $\mathscr{I}_{j}$-closed set. The family $\Gamma_{j}^{\mathscr{I}}$ of all $\mathscr{I}_{j}$-closed sets of a $j$-neighborhood space is defined by $\Gamma_{j}^{\mathscr{I}}=\left\{F \subseteq U: F^{\prime} \in \tau_{j}^{\mathscr{I}}\right\}$.

Definition 9 (see [28]). Let $\left(U, R, \xi_{j}\right)$ be a $j-\mathrm{NS}, A \subseteq U, \mathscr{I}$ an ideal on $U$, and $\forall j \in\{r, l,\langle r\rangle,\langle l\rangle, i, u,\langle i\rangle,\langle u\rangle\}$. The $\mathscr{I}_{j}$-lower, $\mathscr{I}_{j}$-upper approximations, $\mathscr{I}_{j}$-boundary regions, and $\mathscr{I}_{j}$-accuracy of the approximations of $A$ are defined, respectively, as

$$
\begin{aligned}
& \underline{R}_{j}^{\mathscr{I}}(A)=\cup\left\{G \in \tau_{j}^{\mathcal{F}}: G \subseteq A\right\}=\operatorname{int}_{j}^{\mathscr{I}}(A), \quad \text { where int }_{j}^{\mathscr{I}}(A) \text { represents } \mathscr{I}-j-\text { interior of } A \text {, } \\
& \bar{R}_{j}^{\mathcal{F}}(A)=\cap\left\{H \in \Gamma_{j}^{\mathscr{F}}: A \subseteq H\right\}=\mathrm{cl}_{j}^{\mathscr{F}}(A), \quad \text { where } \mathrm{cl}_{j}^{\mathscr{I}}(A) \text { represents } \mathscr{I}-j \text { - closure of } A \text {, } \\
& B_{j}^{\mathcal{F}}(A)=\bar{R}_{j}^{\mathcal{F}}(A)-R_{j}^{\mathcal{F}}(A), \\
& \sigma_{j}^{\mathscr{F}}(A)=\frac{\left|R_{j}^{\mathscr{I}}(A)\right|}{\left|\bar{R}_{j}^{\mathcal{I}}(A)\right|}, \quad \text { where }\left|\bar{R}_{j}^{\mathscr{I}}(A)\right| \neq 0 \text {. }
\end{aligned}
$$

Definition 10 (see [28]). Let $\left(U, R, \xi_{j}\right)$ be a $j-\mathrm{NS}, \mathscr{I}$ an ideal on $U$, and $A \subseteq U, \forall j \in\{r, l,\langle r\rangle,\langle l\rangle, i, u,\langle i\rangle,\langle u\rangle\}$. A subset $A$ is called an $\mathscr{I}$-j-definable $(\mathscr{I}$-j-exact $)$ set if $\bar{R}_{j}^{\mathscr{I}}(A)=R_{j}^{\mathscr{I}}(A)$. Otherwise, $A$ is called an $\mathscr{I}$ - $j$-rough set.

Corollary 1 (see [28]). Let $\left(U, R, \xi_{j}\right)$ be a $j-N S, \mathscr{I}$ an ideal on $U$, and $A \subseteq U$. Then, $\forall j \in\{r, l,\langle r\rangle,\langle l\rangle, i, u,\langle i\rangle,\langle u\rangle\}$.

(1) Every $j$-exact subset in $U$ is $\mathscr{I}$-j-exact

(2) Every $\mathscr{I}$-j-rough subset in $U$ is $j$-rough

\section{Topology Based on Different Neighborhoods by Using Bi-Ideals}

In this section, topologies based on different neighborhoods are generated by using bi-ideals as a generalization of the previous results in $[28,35]$. The main properties of these topologies are studied and compared to the previous ones in $[28,35]$ and shown to be more general.

Theorem 4. Let $\left(U, R, \xi_{j}\right)$ be a $j-N S, A \subseteq U$, and $\mathscr{I}_{1} \vee \mathscr{I}_{2}$ an ideal on $U$. Then, $\forall j \in\{r, l,\langle r\rangle,\langle l\rangle, i, u,\langle i\rangle,\langle u\rangle\}$, and the collection $\tau_{j} \mathcal{\mathscr { F }}_{1} \vee \mathscr{I}_{2}=\left\{A \subseteq U: \forall p \in A, N_{j}(p) \cap A^{\prime} \in \mathscr{I}_{1} \vee \mathscr{I}_{2}\right\}$ is a topology on $U$.
Proof

(1) Clearly, $U$ and $\phi$ belong to $\tau_{j}^{\mathscr{I}_{1} \vee \mathscr{I}_{2}}$.

(2) Let $A_{\delta} \in \tau_{j}^{\mathscr{J}_{1} \vee \mathscr{F}_{2}}(\forall \delta \in \Delta)$ and $a \in \cup_{\delta \in \Delta} A_{\delta}$. Then, $\exists \gamma_{0} \in \Delta$ such that $a \in A_{\delta_{0}}$ :

$$
\begin{aligned}
& \Rightarrow N_{j}(a) \cap A_{\delta_{0}{ }^{\prime} \in \mathscr{I}_{1} \vee \mathscr{I}_{2}} \\
& \Rightarrow N_{j}(a) \cap\left(\cup_{i \in I} A_{\delta}\right)^{\prime} \in \mathscr{I}_{1} \vee \mathscr{I}_{2} \\
& \Rightarrow \cup_{\delta \in \Delta} A_{\delta} \in \tau_{j}^{\mathscr{J}_{1} \vee \mathscr{F}_{2}} .
\end{aligned}
$$

(3) Let $A, B \in \tau_{j}^{\mathscr{F}_{1} \vee \mathscr{F}_{2}}$ and $a \in A \cap B$ :

$$
\begin{aligned}
& \Rightarrow N_{j}(a) \cap A^{\prime} \in \mathscr{I}_{1} \vee \mathscr{I}_{2} \text { and } N_{j}(a) \cap B^{\prime} \in \mathscr{I}_{1} \vee \mathscr{I}_{2} \\
& \Rightarrow\left(N_{j}(a) \cap A^{\prime}\right) \cup\left(N_{j}(a) \cap B^{\prime}\right) \in \mathscr{I}_{1} \vee \mathscr{I}_{2} \\
& \Rightarrow N_{j}(a) \cap\left(A^{\prime} \cup B^{\prime}\right) \in \mathscr{I}_{1} \vee \mathscr{I}_{2} \\
& \Rightarrow\left(N_{j}(a) \cap(A \cap B)^{\prime}\right) \in \mathscr{I}_{1} \vee \mathscr{I}_{2} \\
& \Rightarrow A \cap B \in \tau_{j}^{\mathscr{F}_{1} \vee \mathscr{I}_{2}} .
\end{aligned}
$$

From 1,2 , and $3, \tau_{j}^{\mathscr{J}_{1} \vee \mathscr{F}_{2}}$ is a topology on $U$.

The following theorem shows that the present topologies are than the previous ones in $[28,35]$. 
Theorem 5. Let $\left(U, R, \xi_{j}\right)$ be a $j-N S, A \subseteq U$, and $\mathscr{I}_{1} \vee \mathscr{I}_{2}$ an ideal on $U$. Then, $\forall j \in\{r, l,\langle r\rangle,\langle l\rangle, i, u,\langle i\rangle,\langle u\rangle\}$, $\tau_{j} \subseteq \tau_{j}^{\mathcal{F}_{k}} \subseteq \tau_{j}^{\mathcal{F}_{1} \vee \mathscr{F}_{2}}, k \in\{1,2\}$.

Proof. $\tau_{j} \subseteq \tau_{j}^{\mathscr{F}_{k}}$ by Theorem 3 [28]. To prove $\tau_{j}^{\mathscr{F}_{k}} \subseteq \tau_{j}^{\mathcal{F}_{1} \vee \mathscr{F}_{2}}$, let $A \in \tau_{j}^{\mathscr{F}_{k}}$. Then, $N_{j}(p) \cap A^{\prime} \in \mathscr{I}_{k}, \forall p \in A_{\mathscr{F} \vee \mathscr{F}_{2}}$ Therefore, $N_{j}(p) \cap A_{\mathscr{f}_{1} \vee \mathscr{F}_{2}}^{\prime} \in \mathscr{I}_{1} \vee \mathscr{I}_{2}, \forall p \in A$. So, $A \in \tau_{j}^{\mathscr{F}_{1} \vee \mathscr{F}_{2}}$. Hence,

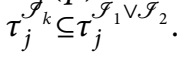

Remark 1. The following points should be noted:

(1) If $\mathscr{I}_{1} \vee \mathscr{I}_{2}=\{\phi\}$ in Theorem 5, then the current generated topologies coincide with the previous one in Theorem 1 [35]. So, the present results are considered as a generalization of Abd El-Monsef et al.'s work [35].

(2) If $\mathscr{I}_{1}=\mathscr{I}_{2}$ in Theorem 5, then the current generated topologies coincide with the previous one in Theorem 2 [28]. So, the present results are an extension of Hosny's work [28].

(3) $\tau_{j} \subsetneq \tau_{j}^{\mathcal{F}_{k}} \subsetneq \tau_{j}^{\mathcal{J}_{1} \vee \mathcal{F}_{2}}$ as shown in the following example.

Example 1. Let $U=\{1,2,3,4\}, R=\{(1,1),(1,2),(1,3)$, $(2,3),(3,4)\}, \mathscr{I}_{1}=\{\phi,\{2\}\}, \mathscr{I}_{2}=\{\phi,\{3\}\}$, and $\mathscr{I}_{1} \vee \mathscr{I}_{2}=$ $\{\phi,\{2\},\{3\},\{2,3\}\}$. The following results are clear:

(1) $\tau_{r}^{\mathcal{F}_{1}}=\{U, \phi,\{4\},\{3,4\},\{1,3,4\},\{2,3,4\}\}, \tau_{r}^{\mathcal{F}_{2}}=\{U, \phi$, $\{2\},\{4\},\{1,2\},\{2,4\},\{3,4\},\{1,2,4\},\{2,3,4\}\}$, and $\tau_{r}^{\mathcal{F}_{1} \vee \mathscr{F}_{2}}=\{U, \phi,\{1\},\{2\},\{4\},\{1,2\},\{1,4\},\{2,4\},\{3,4\}$, $\{1,2,4\},\{1,3,4\},\{2,3,4\}\}$. Thus, $\tau_{r}^{\mathscr{F}_{1}}, \tau_{r}^{\mathcal{F}_{2}} \subsetneq \tau_{r}^{\mathscr{F}_{1} \vee \mathscr{F}_{2}}$.

(2) $\tau_{l}^{\mathcal{F}_{1}}=\{U, \phi,\{1\},\{1,2\},\{1,3\},\{1,2,3\},\{1,3,4\}\}, \tau_{l}^{\mathcal{F}_{2}}=$ $\{U, \phi,\{1\},\{4\},\{1,2\},\{1,4\},\{1,2,3\},\{1,2,4\}\}$, and $\tau_{l}^{\mathcal{F}_{1}} \mathcal{F}_{2}=\{U, \phi,\{1\}, \quad\{4\},\{1,2\},\{1,3\},\{1,4\},\{1,2,3\}$, $\{1,2,4\},\{1,3,4\}\}$. Thus, $\tau_{1}^{\mathcal{F}_{1}}, \tau_{1}^{\gamma_{2}} \subsetneq \tau_{l}^{\mathcal{F}_{1} \vee \mathscr{Y}_{2}}$.

(3) $\tau_{i_{\mathcal{F}}}^{\mathcal{F}_{1}}=P(U)-\{\{1,4\}\}, \tau_{i}^{\mathcal{F}_{2}}=\tau_{i}^{\mathcal{F}_{1} \vee \mathcal{F}_{2}}=P(U)$. Thus,

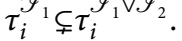

(4) $\tau_{u}^{\mathscr{J}_{1}}=\{U, \phi,\{1,3,4\}\}, \tau_{\mathcal{u}^{\mathcal{F}_{2}}}^{\mathscr{F}}=\{U, \phi,\{4\},\{1,2\},\{1,2,4\}$ \} , and $\tau_{u}^{\mathcal{J}_{1} \vee \mathscr{F}_{2}}=\{U, \phi,\{1\},\{4\},\{1,2\},\{1,4\},\{1,2,4\}$, $\{1,3,4\}\}$. Thus, $\tau_{u}^{\mathcal{F}_{1}}, \tau_{u}^{\mathcal{F}} \subsetneq \tau_{u}^{\mathcal{F}_{1} \vee \mathscr{F}_{2}}$.

(5) $\tau_{\langle\neq\rangle}^{\mathcal{F}_{1}}=\{U, \phi,\{3\},\{4\},\{1,3\},\{3,4\}, \quad\{1,2,3\},\{1,3,4\}\}$, $\tau_{\langle r\rangle}^{\langle(s)}=\{U, \phi,\{3\}, \quad\{4\},\{1,2\},\{3,4\},\{1,2,3\},\{1,2,4\}\}$,

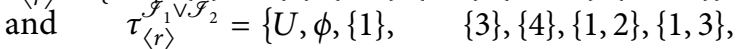
$\{1,4\},\{3,4\},\{1,2,3\},\{1,2,4\}, \quad\{1,3,4\}\} . \quad$ Thus, $\tau_{\langle r\rangle}^{\mathcal{F}_{1}, \tau_{\langle r\rangle} \subsetneq \tau_{\langle r\rangle} \mathcal{F}_{1}, \mathscr{F}_{2}}$.

(6) $\tau_{\langle\mu\rangle}^{\mathscr{J}_{1}}=\{U, \phi,\{3\},\{4\},\{1,3\},\{3,4\},\{1,2,3\},\{1,3,4\}\}$, $\tau_{\langle u\rangle}^{\langle}=\{U, \phi,\{3\},\{4\},\{1,2\},\{3,4\},\{1,2,3\},\{1,2,4\}\}$, and $\tau_{\langle u\rangle}^{\mathcal{F}_{1} \vee \mathcal{Y}_{2}}=\{U, \phi,\{1\},\{3\},\{4\},\{1,2\},\{1,3\},\{1,4\}$, $\{3,4\},\{1,2,3\},\{1,2,4\},\{1,3,4\}\}$. Thus, $\tau_{\langle u\rangle}^{\mathcal{F}_{1}}, \tau_{\langle u\rangle}$ $\subsetneq \tau_{\langle u\rangle}^{\mathcal{F}_{1} \vee \mathcal{Y}_{2}}$.

Similarly, we can add an example to show that

(1) $\tau_{\langle l\rangle}^{\mathscr{F}_{k} \subsetneq \tau_{\langle l\rangle}^{\mathcal{J}_{1} \vee \mathcal{F}_{2}}}$

(2) $\tau_{\langle i\rangle}^{\mathscr{F}_{k} \subsetneq \tau_{\langle i\rangle}^{\mathscr{F}_{1} \vee \mathscr{F}_{2}}}$
Proposition 2. Let $\left(U, R, \xi_{j}\right)$ be a $j-N S$ and $\mathscr{I}_{1} \vee \mathscr{I}_{2}$ an ideal on $U$. Then,

(1) $\tau_{u}^{\mathcal{F}_{1} \vee \mathscr{F}_{2}} \subseteq \tau_{r}^{\mathcal{F}_{1} \vee \mathscr{F}_{2}}$ and $\tau_{u}^{\mathcal{F}_{1} \vee \mathcal{F}_{2}} \subseteq \tau_{l}^{\mathcal{F}_{1} \vee \mathscr{F}_{2}}$

(2) $\tau_{r}^{\mathscr{F}_{1} \vee \mathscr{F}_{2}} \subseteq \tau_{i}^{\mathcal{F}_{1} \vee \mathscr{F}_{2}}$ and $\tau_{1}^{\mathcal{F}_{1} \vee \mathscr{F}_{2}} \subseteq \tau_{i}^{\mathscr{F}_{1} \vee \mathscr{F}_{2}}$

(3) $\tau_{\langle u\rangle}^{\mathcal{J}_{1} \vee \mathscr{F}_{2}} \subseteq \tau_{\langle r\rangle}^{\mathcal{J}_{1} \vee \mathscr{F}_{2}}$ and $\tau_{\langle u\rangle}^{\mathcal{G}} \subseteq \tau_{\langle l\rangle}^{\mathscr{J}} \vee \mathscr{F}_{2}$

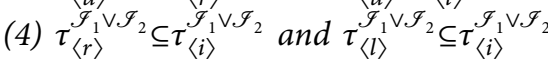

Proof

(1) Let $\quad A \in \tau_{u}^{\mathscr{J}_{1} \vee \mathscr{F}_{2}}$. Then, $\quad N_{u}(p) \cap A^{\prime} \in \mathscr{I}_{1} \vee \mathscr{I}_{2}$, $\forall p \in A$. Thus, $\quad\left(N_{r}(p) \cup N_{l}(p)\right) \cap A^{\prime} \in \mathscr{I}_{1} \vee \mathscr{I}_{2}$, $\forall p \in A$. Hence, $N_{r}(p) \cap A^{\prime} \in \mathscr{I}_{1} \vee \mathscr{I}_{2}, \forall p \in A$ and $N_{l}(p) \cap A^{\prime} \in \mathscr{I}_{1} \vee \mathscr{F}_{2}, \forall p \in A$. Therefore, $A \in$

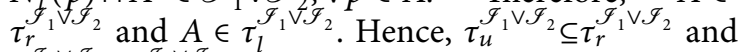
$\tau_{\mathcal{u}}^{\mathcal{J}_{1} \vee \mathscr{V}_{2}} \subseteq \tau_{l}^{\mathcal{F}_{1} \vee \mathscr{F}_{2}}$. Similarly, we can prove 3 .

(2) Let $A \in \tau_{r}^{\mathscr{F}_{1} \vee \mathscr{F}_{2}}$. Then, $N_{r}(p) \cap A^{\prime} \in \mathscr{I}_{1} \vee \mathscr{I}_{2}$, $\forall p \in A$. Thus, $\left(N_{r}(p) \cap N_{l}(p)\right) \cap A^{\prime} \in \mathscr{I}_{1} \vee \mathscr{I}_{2}, \forall p$ $\in A$. Hence, $N_{i}(p) \cap A^{\prime} \in \mathscr{I}_{1} \vee \mathscr{I}_{2}, \forall p \in A$. Therefore, $A \in \tau_{i}^{\mathcal{F}_{1} \vee \mathcal{F}_{2}}$. Hence, $\tau_{r}^{\mathcal{F}_{1}} \mathcal{F}_{2} \subseteq \tau_{i}^{\mathcal{F}_{1} \vee \mathcal{F}_{2}}$. Similarly, we can prove 4 .

Corollary 2. Let $\left(U, R, \xi_{j}\right)$ be a $j-N S$ and $\mathscr{I}_{1} \vee \mathscr{I}_{2}$ an ideal on $U$. Then, $\forall j \in\{r, l,\langle r\rangle,\langle l\rangle, i, u,\langle i\rangle,\langle u\rangle\}$.

(1) $\tau_{u}^{\mathscr{J}_{1} \vee \mathscr{I}_{2}} \subseteq \tau_{r}^{\mathcal{F}_{1} \vee \mathscr{I}_{2}} \subseteq \tau_{i}^{\mathcal{F}_{1} \vee \mathscr{F}_{2}}$

(2) $\tau_{u}^{\mathcal{F}_{1} \vee \mathscr{F}_{2}} \subseteq \tau_{l}^{\mathcal{F}_{1} \vee \mathscr{F}_{2}} \subseteq \tau_{i}^{\mathcal{F}_{1} \vee \mathscr{F}_{2}}$

(3) $\tau_{\langle u\rangle}^{\mathscr{J}_{1} \vee \mathscr{I}_{2}} \subseteq \tau_{\langle r\rangle}^{\mathscr{J}_{1} \vee \mathscr{I}_{2}} \subseteq \tau_{\langle i\rangle}^{\mathscr{J}_{1} \vee \mathscr{F}_{2}}$

(4) $\tau_{\langle u\rangle}^{\mathscr{F}_{1} \vee \mathscr{F}_{2}} \subseteq \tau_{\langle l\rangle}^{\mathscr{F}_{1} \vee \mathscr{F}_{2}} \subseteq \tau_{\langle i\rangle}^{\mathcal{F}_{1} \vee \mathscr{F}_{2}}$

Remark 2

(i) Example 1 shows that the inclusion in Proposition 2 and Corollary 2 cannot be replaced by equality relation as follows:

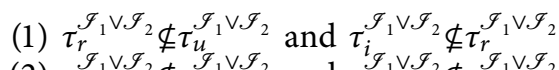

(2) $\tau_{l}^{\mathscr{F}_{1} \vee \mathscr{F}_{2} \nsubseteq \tau_{\mathcal{u}} \mathcal{I} \vee \mathscr{F}_{2}}$ and $\tau_{i}^{\mathcal{F}_{1} \vee \mathscr{F}_{2}} \nsubseteq \tau_{l}^{\mathcal{F}_{1} \vee \mathscr{F}_{2}}$

(ii) Similarly, we can add an example to show that

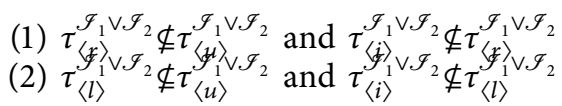

Remark 3. Let $\left(U, R, \xi_{j}\right)$ be a $j-\mathrm{NS}$ and $\mathscr{I}_{1} \vee \mathscr{I}_{2}$ an ideal on $U$. Then, the following statements should be noted:

(1) $\tau_{r}^{\mathscr{F}_{1} \vee \mathscr{F}_{2}}$ is not the dual of $\tau_{l}^{\mathcal{F}_{1} \vee \mathscr{F}_{2}}$ (see Example 1). Although $\tau_{r}$ is the dual of $\tau_{l}$, it is proved in [35].

(2) $\tau_{r}^{\mathcal{F}_{1} \vee \mathcal{F}_{2}}$ and $\tau_{\langle r\rangle}^{\mathcal{F}_{1} \vee \mathcal{F}_{2}}$ are not necessarily to be comparable (see Example 1).

(3) $\tau_{l}^{\mathcal{F}_{1} \vee \mathscr{F}_{2}}$ and $\tau_{\langle l\rangle}^{\mathcal{F}_{1} \vee \mathcal{F}_{2}}$ are not necessarily to be comparable (see Example 1).

(4) $\tau_{i}^{\mathcal{F}_{1} \vee \mathcal{F}_{2}}$ and $\tau_{\langle i\rangle}^{\mathcal{F}_{1} \vee \mathscr{F}_{2}}$ are not necessarily to be comparable. 
(5) If $R$ is a reflexive relation, then the following topologies are comparable as follows:
(a) $\tau_{\langle r\rangle}^{\mathscr{G} \vee \mathscr{F}_{2} \subseteq \tau_{r}^{\mathscr{J}}{ }^{\mathcal{F}} \vee \mathcal{F}_{2}}$
(b) $\tau_{\langle l\rangle}^{\mathcal{J}_{1} \vee \mathscr{F}_{2}} \subseteq \tau_{l}^{\mathcal{J}_{1} \vee \mathscr{F}_{2}}$
(c) $\tau_{\langle i\rangle}^{\mathscr{I}_{1} \vee \mathscr{F}_{2}} \subseteq \tau_{i}^{\mathscr{J}_{1} \vee \mathscr{F}_{2}}$

Definition 11. Let $\left(U, R, \xi_{j}\right)$ be a $j-\mathrm{NS}$ and $\mathscr{I}_{1} \vee \mathscr{I}_{2}$ an ideal on $U$. A subset $A \subseteq U$ is called an $\mathscr{I}_{1} \vee \mathscr{I}_{2} j^{\text {-open set if }}$ $A \in \tau_{j}^{\mathscr{F}_{1} \vee \mathscr{F}_{2}}$ and the complement of an $\mathscr{I}_{1} \vee \mathscr{I}_{2}$-open set is called an $\mathscr{I}_{1} \vee \mathscr{I}_{2 j}$-closed set. The family $\Gamma_{j}^{\mathscr{F}_{1} \vee \mathscr{F}_{2}}$ of all $\mathscr{I}_{1} \vee \mathscr{I}_{2}$-closed sets of a $j$-neighborhood space is defined by $\Gamma_{j}^{\mathcal{J}_{1} \vee \mathscr{F}_{2}}=\left\{F \subseteq U: F^{\prime} \in \tau_{j}^{\mathcal{F}_{1} \vee \mathscr{F}_{2}}\right\}$.

Theorem 6. Let $\left(U, R, \xi_{j}\right)$ be a $j-N S$ and $\mathscr{I}_{1} \vee \mathscr{I}_{2}, \mathscr{J}_{1} \vee \mathscr{F}_{2}$ two ideals on $U$. If $\mathscr{I}_{1} \vee \mathscr{I}_{2} \subseteq \mathscr{F}_{1} \vee \mathscr{F}_{2}$, then $\forall j \in\{r, l,\langle r\rangle,\langle l\rangle, i, u,\langle i\rangle,\langle u\rangle\}$,

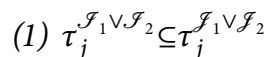

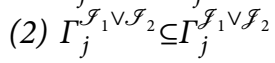

Proof

(1) Let $A \in \tau_{j}^{\mathscr{F}_{1} \vee \mathscr{F}_{2}} ; \quad$ then, $\quad \forall p \in A, N_{j}(p) \cap A^{\prime} \in$ $\mathscr{I}_{1} \vee \mathscr{I}_{2} \subseteq \mathscr{F}_{1} \vee \mathscr{F}_{2}$. Thus, $\forall p \in A, N_{j}(p) \cap A^{\prime} \in \mathscr{F}_{1} \vee$

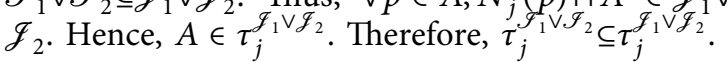

(2) Immediately from (1).

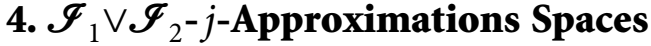

In this section, more general notions of approximations are suggested. One of these notions is based on the union of two lower approximations and the intersections of the two upper approximations. On the other hand, there exists a close relationship between topologies and rough sets. So, the other notions explore rough set theory from the point of view of topology.

Definition 12. Let $\left(U, R, \xi_{j}\right)$ be a $j-\mathrm{NS}, A \subseteq U, \mathscr{J}_{k}, \forall k \in\{1,2\}$ two ideals on $U$, and $\forall j \in\{r, l,\langle r\rangle,\langle l\rangle, i, u,\langle i\rangle,\langle u\rangle\}$. The $\mathscr{I}_{1}, \mathscr{I}_{2}$-j-lower, $\quad \mathscr{I}_{1}, \mathscr{I}_{2}$-j-upper $\quad$ approximations, $\mathscr{I}_{1}, \mathscr{I}_{2}$-j-boundary regions, and $\mathscr{I}_{1}, \mathscr{I}_{2}$-j-accuracy of the approximations of $A$ are defined, respectively, as

$R_{j}^{\mathscr{F}_{1}, \mathscr{F}_{2}}(A)=R_{j}^{\mathscr{J}_{1}}(A) \cup R_{j}^{\mathscr{F}_{2}}(A)$, where $R_{j}^{\mathscr{F}_{k}}(A)$ is the $\mathscr{I}_{k^{-}}$-jower approximations of $A$ with respect to $\mathscr{I}_{k}, k \in\{1,2\}$ as in Definition 9

$\bar{R}_{j}^{\mathcal{F}_{1}, \mathcal{F}_{2}}(A)=\bar{R}_{j}^{\mathscr{F}_{1}}(A) \cap \bar{R}_{j}^{\mathscr{F}^{2}}(A)$, where $\bar{R}_{j}^{\mathscr{F}_{k}}(A)$ is the $\mathscr{I}_{k}$-j-upper approximations of $A$ with respect to $\mathscr{J}_{k}, k \in\{1,2\}$ as in Definition 9

$$
\begin{aligned}
& B_{j}^{\mathcal{F}_{1}, \mathscr{F}_{2}}(A)=\bar{R}_{j}^{\mathscr{F}_{1}, \mathscr{F}_{2}}(A)-R_{j}^{\mathscr{J}_{1}, \mathscr{F}_{2}}(A)
\end{aligned}
$$

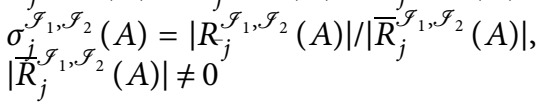

where

Proposition 3. Let $\left(U, R, \xi_{j}\right)$ be a j-NS, $A, B \subseteq U, \mathscr{I}_{1}, \mathscr{I}_{2}$ two ideals on $U$, and $\forall j \in\{r, l,\langle r\rangle,\langle l\rangle, i, u,\langle i\rangle,\langle u\rangle\}$. Then, the following properties hold:
(1) $R_{j}^{\mathcal{F}_{1}, \mathscr{F}_{2}}(A) \subseteq A \subseteq \bar{R}_{j}^{\mathscr{F}_{1}, \mathscr{F}_{2}}(A) R_{j}^{\mathscr{F}_{1}, \mathscr{F}_{2}}(A) \subseteq A \subseteq \bar{R}_{j}^{\mathscr{F}_{1}, \mathscr{F}_{2}}(A)$; equality holds if $A=\phi$ or $U$

(2) $A \subseteq B \Rightarrow \bar{R}_{j}^{\mathcal{F}_{1}, \mathscr{F}_{2}}(A) \subseteq \bar{R}_{j}^{\mathcal{F}_{1}, \mathscr{F}_{2}}(B)$ and $R_{j}^{\mathcal{F}_{1}, \mathscr{F}_{2}}(A) \subseteq$ $R_{j}^{\mathscr{J}_{1}, \mathscr{F}_{2}}(B)$

(3) $\bar{R}_{j}^{\mathcal{F}_{1}, \mathscr{F}_{2}}(A \cap B) \subseteq \bar{R}_{j}^{\mathscr{F}_{1}, \mathscr{F}_{2}}(A) \cap \bar{R}_{j}^{\mathscr{F}_{1}, \mathscr{F}_{2}}(B)$

(4) $\bar{R}_{j}^{\mathcal{F}_{1}, \mathscr{F}_{2}}(A \cup B) \supseteq \bar{R}_{j}^{\mathcal{J}_{1}, \mathscr{F}_{2}}(A) \cup \bar{R}_{j}^{\mathcal{F}_{1}, \mathscr{F}_{2}}(B)$

(5) $R_{j}^{\mathcal{J}_{1}, \mathscr{F}_{2}}(A \cap B) \subseteq R_{j}^{\mathcal{J}_{1}, \mathscr{F}_{2}}(A) \cap R_{j}^{\mathscr{F}_{1}, \mathscr{F}_{2}}(B)$

(6) $R_{j}^{\mathcal{F}_{1}, \mathscr{F}_{2}}(A \cup B) \supseteq R_{j}^{\mathcal{F}_{1}, \mathscr{F}_{2}}(A) \cup R_{j}^{\mathscr{F}_{1}, \mathscr{F}_{2}}(B)$

(7) $\bar{R}_{j}^{\mathcal{J}_{1}, \mathscr{F}_{2}}(A)=\left[R_{j}^{\mathcal{F}_{1}, \mathscr{F}_{2}}\left(A^{\prime}\right)\right]^{\prime}, R_{j}^{\mathcal{F}_{1}, \mathscr{F}_{2}}(A)=\left[\bar{R}_{j}^{\mathcal{F}_{1}, \mathscr{F}_{2}}\right.$ $\left.\left(A^{\prime}\right)\right]^{\prime}$

Proof. The proof is straightforward by using Definitions 12 and 9 [28].

Remark 4. Example 1 shows that

(1) The inclusion in parts 1, 3, 4, 5, and 6 of Proposition 3 cannot be replaced by equality relation:

(i) For part 1 , if $A=\{1,4\}$, then $R_{r}^{\mathcal{F}_{1}, \mathscr{F}_{2}}$ $(A)=\{4\} \neq\{1,4\}=A \neq\{1,3,4\}=\bar{R}_{r}^{\mathscr{F}_{1}}, \mathscr{I}_{2}(A)$

(ii) For ${ }_{A}$ part 3 , if $A=\{1,3\}, B=\{1,4\}$, then $\bar{R}_{r_{\mathscr{F}_{1}}, \mathcal{F}_{2}}^{\mathcal{F}_{1}}(A \cap B)=\{1\} \neq\{1,3\}=\left(\bar{R}_{r}^{\mathcal{F}_{1}, \mathscr{F}_{2}}(A) \cap\right.$ $\left.\bar{R}_{r}^{\mathcal{F}_{1}, \mathscr{F}_{2}}(B)\right)$

(iii) For part 4 , if $A=\{2\}, B=\{3\}$, then $\bar{R}_{r}^{\mathcal{J}_{1}, \mathscr{F}_{2}}$ $(A \cup B)=\{1,2,3\} \neq\{2,3\}=\left(\bar{R}_{r}^{\mathscr{F}_{1}, \mathcal{F}_{2}}(A) \cup\right.$ $\left.\bar{R}_{r}^{\mathcal{Y}_{1}, \mathscr{G}_{2}}(B)\right)$

(iv) For part 5, if $A=\{1,2,4\}, B=\{1,3,4\}$, then $R_{r_{r}, \mathscr{I}_{2}}^{\mathscr{F}_{1}}(A \cap B)=\{4\} \neq\{1,4\} \quad=\left(R_{r}^{\mathscr{F}_{1}, \mathscr{F}_{2}}(A) \cap\right.$ $\left.R_{r}^{\mathscr{F}_{1}, \mathscr{F}_{2}}(B)\right)$

(v) For part 6, if $A=\{1,3\}, B=\{1,4\}$, then $R_{r}^{\mathscr{J}_{1}, \mathscr{F}_{2}}(A \cup B)=\{1,3,4\} \neq\{4\}=\left(R_{r}^{\mathscr{J}_{1}}\right.$, $\left.\mathscr{I}_{2}(A) \cup R_{r}^{\mathscr{F}_{1}}, \mathscr{I}_{2}(B)\right)$

(2) The converse of part 2 of Proposition 3 is not necessarily true; if $A=\{3\}, B=\{4\}$, then $\bar{R}_{r}^{\mathscr{J}_{1}, \mathscr{Y}_{2}}(A)=$ $\{3\} \quad \subseteq\{3,4\}=\bar{R}_{r}^{\mathscr{F}_{1}, \mathscr{F}_{2}}(B)$ and $R_{r}^{\mathscr{J}_{1}, \mathscr{F}_{2}}(A)=$ $\phi \subseteq\{4\}=R_{r}^{\mathcal{I}_{1}, \mathscr{F}_{2}}(B)$, but $A \nsubseteq B$

Definition 13. Let $\left(U, R, \xi_{j}\right)$ be a $j$-NS and $\mathscr{I}_{1}, \mathscr{I}_{2}$ two ideals on $U, A \subseteq U, \forall j \in\{r, l,\langle r\rangle,\langle l\rangle, i, u,\langle i\rangle,\langle u\rangle\}$. A subset $A$ is called an $\mathscr{I}_{1}, \mathscr{I}_{2}$-j-definable (an $\mathscr{I}_{1}, \mathscr{I}_{2}$-j-exact) set if $\bar{R}_{j} \mathcal{F}_{1}, \mathscr{F}_{2}(A)=R_{j}^{\mathcal{F}_{1}, \mathscr{F}_{2}}(A)$. Otherwise, $A$ is called an $\mathscr{I}_{1}, \mathscr{I}_{2}$-j-rough set.

Relationships between Abd El-Monsef et. al's approximations in Definition 6 [28], Hosny's approximations in Definition 9 [35], and the present approximations in Definition 12 are given in the following proposition.

Proposition 4. Let $\left(U, R, \xi_{j}\right)$ be a j-NS, $A \subseteq U, \mathscr{I}_{k}, \forall k \in\{1,2\}$ two ideals on $U$, and $\forall j \in\{r, l,\langle r\rangle,\langle l\rangle, i, u,\langle i\rangle,\langle u\rangle\}$. Then,

(1) $R(A) \subseteq R_{j}^{\mathscr{J}_{k}}(A) \subseteq R_{j}^{\mathcal{J}_{1}, \mathscr{F}_{2}}(A)$

(2) $\bar{R}_{j}^{j \mathscr{R}_{1}, \mathscr{F}_{2}}(A) \subseteq \bar{R}_{j}^{\mathscr{F}_{k}}(A) \subseteq \bar{R}_{j}(A)$

(3) $B_{j}^{\mathscr{F}_{1}, \mathscr{F}_{2}}(A) \subseteq B_{j}^{\mathscr{F}_{k}}(A) \subseteq B_{j}(A)$

(4) $\sigma_{j}(A) \leq \sigma_{j}^{\mathcal{F}_{k}}(A) \leq \sigma_{j}^{\mathscr{J}_{1}, \mathscr{F}_{2}}(A)$ 
Proof. Immediately by using Definitions 12, 6 [35] and 9 [28].

Definition 14. Let $\left(U, R, \xi_{j}\right)$ be a $j-\mathrm{NS}, A \subseteq U, \mathscr{I}_{1} \vee \mathscr{I}_{2}$ an ideal on $U$, and $\forall j \in\{r, l,\langle r\rangle,\langle l\rangle, i, u,\langle i\rangle,\langle u\rangle\}$. The
$\mathscr{I}_{1} \vee \mathscr{I}_{2}$-j-lower, $\quad \mathscr{I}_{1} \vee \mathscr{I}_{2}$-j-upper approximations, $\mathscr{I}_{1} \vee \mathscr{I}_{2}$-j-boundary regions, and $\mathscr{I}_{1} \vee \mathscr{I}_{2}$-j-accuracy of the approximations of $A$ are defined, respectively, as

$$
\begin{aligned}
& \underline{R}_{j}^{\mathscr{F}_{1} \vee \mathscr{F}_{2}}(A)=\cup\left\{G \in \tau_{j}^{\mathscr{F}_{1} \vee \mathscr{F}_{2}}: G \subseteq A\right\}=\operatorname{int}_{j}^{\mathcal{F}_{1} \vee \mathscr{F}_{2}}(A), \quad \text { where int }{ }_{j}^{\mathcal{F}_{1} \vee \mathscr{F}_{2}}(A) \text { represents } \mathscr{I}_{1} \vee \mathscr{I}_{2}-j-\text { interior of } A, \\
& \bar{R}_{j}^{\mathscr{F}_{1} \vee \mathscr{F}_{2}}(A)=\cap\left\{H \in \Gamma_{j}^{\mathscr{I}_{1} \vee \mathscr{F}_{2}}: A \subseteq H\right\}=\mathrm{cl}_{j}^{\mathscr{F}_{1} \vee \mathscr{F}_{2}}(A), \quad \text { where cl }{ }_{j}^{\mathscr{I}_{1} \vee \mathscr{F}_{2}}(A) \text { represents } \mathscr{I}_{1} \vee \mathscr{I}_{2}-j-\text { interior of } A \text {, } \\
& B_{j}^{\mathcal{F}_{1} \vee \mathscr{F}_{2}}(A)=\bar{R}_{j}^{\mathscr{F}_{1} \vee \mathscr{F}_{2}}(A)-R_{j}^{\mathcal{F}_{1} \vee \mathscr{F}_{2}}(A), \\
& \sigma_{j}^{\mathscr{F}_{1} \vee \mathscr{F}_{2}}(A)=\frac{\left|R_{j}^{\mathscr{F}_{1} \vee \mathscr{F}_{2}}(A)\right|}{\left|\bar{R}_{j}^{\mathscr{F}_{1} \vee \mathscr{I}_{2}}(A)\right|}, \quad \text { where }\left|\bar{R}_{j}^{\mathscr{F}_{1} \vee \mathscr{F}_{2}}(A)\right| \neq 0 .
\end{aligned}
$$

The basic properties of the current $\mathscr{I}_{1} \vee \mathscr{I}_{2}$ - $j$-lower and $\mathscr{I}_{1} \vee \mathscr{I}_{2}$-j-upper approximations are introduced in the following proposition.

Proposition 5. Let $\left(U, R, \xi_{j}\right)$ be a $j-N S, \mathscr{I}_{1} \vee \mathscr{I}_{2}$ an ideal on $U$, and $A, B \subseteq U$. Then, $\forall j \in\{r, l,\langle r\rangle,\langle l\rangle, i, u,\langle i\rangle,\langle u\rangle\}$ :

(1) $R_{j}^{\mathscr{F}_{1} \vee \mathscr{F}_{2}}(A) \subseteq A \subseteq \bar{R}_{j}^{\mathscr{J}_{1} \vee \mathscr{F}_{2}}(A)$ equality holds if $A=\phi$
or $U$

(2) $A \subseteq B \Rightarrow \bar{R}_{j}^{\mathscr{I}_{1} \vee \mathscr{I}_{2}}(A) \subseteq \bar{R}_{j}^{\mathcal{F}_{1} \vee \mathscr{I}_{2}}(B)$

(3) $A \subseteq B \Rightarrow R_{j}^{\mathscr{F}_{1} \vee \mathscr{F}_{2}}(A) \subseteq R_{j}^{\mathscr{I}_{1} \vee \mathscr{I}_{2}}(B)$

(4) $\bar{R}_{j}^{\mathscr{I}_{1} \vee \mathscr{I}_{2}}(A \cap B) \subseteq \bar{R}_{j}^{\mathscr{F}_{1} \vee \mathscr{F}_{2}}(A) \cap \bar{R}_{j}^{\mathscr{I}_{1} \vee \mathscr{I}_{2}}(B)$

(5) $R_{j}^{\mathscr{J}_{1} \vee \mathscr{F}_{2}}(A \cup B) \supseteq R_{j}^{\mathscr{J}_{1} \vee \mathscr{F}_{2}}(A) \cup R_{j}^{\mathscr{F}_{1} \vee \mathscr{F}_{2}}(B)$

(6) $\bar{R}_{j}^{\mathscr{F}_{1} \vee \mathscr{I}_{2}}(A \cup B)=\bar{R}_{j}^{\mathscr{I}_{1} \vee \mathscr{F}_{2}}(A) \cup \bar{R}_{j}^{\mathscr{J}_{1} \vee \mathscr{F}_{2}}(B)$

(7) $R_{j}^{\mathscr{F}_{1} \vee \mathscr{F}_{2}}(A \cap B)=R_{j}^{\mathscr{F}_{1} \vee \mathscr{I}_{2}}(A) \cap R_{j}^{\mathscr{F}_{1} \vee \mathscr{F}_{2}}(B)$

(8) $R_{j_{\mathcal{F}_{1}} \vee \mathscr{F}_{2}}^{\mathscr{F}}(A)=\left(\bar{R}_{j_{1} \vee \mathscr{F}_{2}}^{\mathcal{J}_{1}}\left(A^{\prime}\right)\right)^{\prime}$ $\bar{R}_{j}^{j_{F_{1}} \vee \mathscr{F}_{2}}(A)=\left(R_{j}^{j_{\mathcal{F}_{1}} \vee \mathscr{I}_{2}}\left(A^{\prime}\right)\right)^{\prime}$

(9) $\bar{R}_{j}^{\mathscr{J}_{1} \vee \mathscr{I}_{2}}\left(\bar{R}_{j}^{\mathscr{J}_{1} \vee \mathscr{I}_{2}}(A)\right)=\bar{R}_{j}^{\mathscr{J}_{1} \vee \mathscr{F}_{2}}(A)$

(10) $R_{j}^{\mathscr{F}_{1} \vee \mathscr{F}_{2}}\left(R_{j}^{\mathscr{I}_{1} \vee \mathscr{F}_{2}}(A)\right)=R_{j}^{\mathscr{F}_{1} \vee \mathscr{I}_{2}}(A)$

(11) $R_{j}^{\mathscr{F}_{1} \vee \mathscr{F}_{2}}\left(R_{j}^{\mathscr{F}_{1} \vee \mathscr{F}_{2}}(A)\right) \subseteq \bar{R}_{j}^{\mathscr{I}_{1} \vee \mathscr{F}_{2}}\left(R_{j}^{\mathscr{I}_{1} \vee \mathscr{F}_{2}}(A)\right)$

(12) $R_{j}^{\mathscr{I}_{1} \vee \mathscr{I}_{2}}\left(\bar{R}_{j}^{\mathcal{F}_{1} \vee \mathscr{I}_{2}}(A)\right) \subseteq \bar{R}_{j}^{\mathscr{I}_{1} \vee \mathscr{I}_{2}}\left(\bar{R}_{j}^{\mathcal{F}_{1} \vee \mathscr{I}_{2}}(A)\right)$

The proof of this proposition is simple using the properties of $\mathscr{I}_{1} \vee \mathscr{I}_{2}$-j-interior and $\mathscr{I}_{1} \vee \mathscr{I}_{2}$-j-closure, so we omit it.

Remark 5. Example 1 shows the following:

(1) The inclusion in parts $1,4,5,11$, and 12 of Proposition 5 cannot be replaced by equality relation (take $j=r$ ):

(i) For part 1, if $A=\{3\}, R_{r}^{\mathcal{F}_{1} \vee \mathscr{I}_{2}}(A)=\phi$, then $A \nsubseteq R_{r}^{\mathscr{I}_{1} \vee \mathscr{I}_{2}}(A)$. If $A=\{4\}, \bar{R}_{r}^{\mathscr{I}_{1} \vee \mathscr{F}_{2}}(A)=\{3,4\}$, then $\bar{R}_{r}^{\mathcal{I}_{1} \vee \mathscr{F}_{2}}(A) \nsubseteq A$. (ii) For part 4, if $A=\{3\}, B=\{4\}, A \cap B=\phi, \bar{R}$ ${ }_{r}^{\mathscr{I}_{1} \vee \mathscr{F}_{2}}(A)=\{3\}, \bar{R}_{r}^{\mathscr{F}_{1}} \quad \vee \mathscr{I}_{2}(B)=\{3,4\}, \bar{R}_{r_{1}}^{\mathscr{F}_{1}} \mathscr{\mathscr { F }}_{2}$ $(A \cap B)=\phi$, then $\bar{R}_{r}^{\mathscr{F}_{1}^{2} \vee \mathscr{F}_{2}}(A) \cap \bar{R}_{r}^{\mathscr{F}_{1} \vee \mathscr{F}_{2}}(B)$ $=\{3\} \nsubseteq \phi=\bar{R}_{r} \mathscr{F}_{1} \vee \mathscr{I}_{2}(A \cap B)$.

(iii) For part $5, \quad$ if $A=\{3\}, B=\{4\}$, $A \cup B=\{3,4\}, R_{r}^{\mathscr{I}_{1} \vee \mathscr{I}_{2}}(A)=\phi, R_{r}^{\mathscr{I}_{1}} \quad \vee \mathscr{I}_{2}(B)=$ $\{4\}, R_{r}^{\mathscr{I}_{1} \vee \mathscr{I}_{2}}(A \cup B)=\{3,4\}$, then $R_{r}^{\mathscr{I}_{1} \vee \mathscr{I}_{2}}$ $(A \cup B)=\{3,4\} \nsubseteq\{4\}=R_{r}^{\mathscr{I}_{1} \vee \mathscr{I}_{2}}(A) \cup R_{r}^{\mathscr{I}_{1} \vee \mathscr{I}_{2}}$ (B).

(iv) For part 11, if $A=\{4\}, R_{r}^{\mathscr{I}_{1} \vee \mathscr{F}_{2}}\left(R_{r}^{\mathscr{F}_{1} \vee \mathscr{F}_{2}}(A)\right)=$ $A, \bar{R}_{\mathscr{F}_{1} \vee \mathscr{I}_{2}}\left(R_{r}^{\mathscr{I}_{1} \vee} \quad \mathscr{I}_{2}(A)\right)=\{3,4\}$, then $\bar{R}_{r}^{\mathscr{F}_{1} \vee \mathscr{I}_{2}}\left(R_{r}^{\mathscr{I}_{1} \vee \mathscr{I}_{2}}(A)\right) \nsubseteq R_{r}^{\mathscr{I}_{1} \vee \mathscr{I}_{2}}\left(R_{r}^{\mathscr{I}_{1} \vee \mathscr{I}_{2}}(A)\right)$.

(v) For part 12, if $A=\{3\}, \bar{R}_{r}^{\mathcal{F}_{1} \vee \mathscr{F}_{2}^{r}}\left(\bar{R}_{r}^{\mathcal{F}_{1} \vee \mathscr{F}_{2}}(A)\right)=$ $A, R_{r}^{\mathscr{I}_{1} \vee \mathscr{I}_{2}} \quad\left(\bar{R}_{r}^{\mathscr{J}_{1} \vee \mathscr{I}_{2}}(A)\right)=\phi_{r}$, then $\bar{R}_{r}^{A}{ }_{\mathscr{F}_{1}}{ }^{\mathscr{F}_{2}}\left(\bar{R}_{r}^{\mathscr{J}_{1} \vee \mathscr{I}_{2}}(A)\right) \nsubseteq R_{r}^{\mathscr{I}_{1} \vee \mathscr{I}_{2}}\left(\bar{R}_{r}^{\mathscr{F}_{1} \vee \mathscr{I}_{2}}(A)\right)$.

(2) The converse of parts 2 and 3 of Proposition 5 is not necessarily true (take $j=r$ ):

(i) For part 2, if $A=\{3\}, B=\{4\}$, then $\bar{R}_{r_{1} \vee \mathscr{I}_{2}}(A)=\{3\}, \bar{R}_{r}^{\mathscr{I}_{1} \vee \mathscr{I}_{2}}(B)=\{3,4\}$. Therefore, $\bar{R}_{r}^{r_{\mathcal{F}_{1}} \vee \mathscr{F}_{2}}(A) \subseteq \bar{R}_{r} \mathscr{F}_{1} \vee \mathscr{F}_{2}^{r}(B)$, but $A \nsubseteq B$.

(ii) For part 3, if $A=\{3\}, B=\{2\}$, then $R_{r}^{\mathscr{J}_{1} \vee \mathscr{I}_{2}}(A)=\phi, R_{r}^{\mathscr{I}_{1} \vee \mathscr{I}_{2}}(B)=\{2\}$. Therefore, $R_{r}^{\mathscr{F}_{1} \vee \mathscr{I}_{2}}(A) \subseteq R_{r}^{\mathscr{I}_{1} \vee \mathscr{I}_{2}}(B)$, but $A \nsubseteq B$.

Definition 15. Let $\left(U, R, \xi_{j}\right)$ be a $j-\mathrm{NS}, \mathscr{I}_{1} \vee \mathscr{I}_{2}$ an ideal on $U, A \subseteq U$, and $\forall j \in\{r, l,\langle r\rangle,\langle l\rangle, i, u,\langle i\rangle,\langle u\rangle\}$. A subset $A$ is called an $\mathscr{I}_{1} \vee \mathscr{I}_{2}$-j-definable (an $\mathscr{I}_{1} \vee \mathscr{I}_{2}$-j-exact) set if $\bar{R}_{j}^{\mathscr{F}_{1} \vee \mathscr{F}_{2}}(A)=R_{j}^{\mathscr{F}_{1} \vee \dot{F}_{2}}(A)$. Otherwise, $A$ is called an $\mathscr{I}_{1} \vee \mathscr{I}_{2}$-j-rough set.

In Example $1, A=\{2\}$ is $\mathscr{I}_{1} \vee \mathscr{I}_{2}-r$-exact, while $B=\{3\}$ is $\mathscr{I}_{1} \vee \mathscr{I}_{2}$-r-rough.

Remark 6. Let $\left(U, R, \xi_{j}\right)$ be a $j-\mathrm{NS}, \mathscr{I}_{1} \vee \mathscr{I}_{2}$ an ideal on $U$, and $A \subseteq U$. Then, $\forall j \in\{r, l,\langle r\rangle,\langle l\rangle, i, u,\langle i\rangle,\langle u\rangle\}$, the intersection of two $\mathscr{I}_{1} \vee \mathscr{I}_{2}$-j-rough sets need not be an $\mathscr{I}_{1} \vee \mathscr{I}_{2}$-j-rough set as in Example $1,\{1,3\}$ and $\{1,4\}$ are 
$\mathscr{I}_{1} \vee \mathscr{I}_{2}$-r-rough sets, and $\{1,3\} \cap\{1,4\}=\{1\}$ is not an $\mathscr{I}_{1} \vee \mathscr{I}_{2}-r$-rough set.

The following theorem and corollary present the comparisons between the present approximations in Definitions 12 and 14 and the previous ones in Definitions 6 [35] and 9 [28].

Theorem 7. Let $\left(U, R, \xi_{j}\right)$ be a $j-N S, \mathscr{I}_{1} \vee \mathscr{I}_{2}$ an ideal on $U$, and $A \subseteq U$. Then, $\forall j \in\{r, l,\langle r\rangle,\langle l\rangle, i, u,\langle i\rangle,\langle u\rangle\}$ and $\forall k \in\{1,2\}$.
(1) $R(A) \subseteq R_{j}^{\mathcal{F}_{k}}(A) \subseteq R_{j}^{\mathscr{J}_{1}, \mathscr{F}_{2}}(A) \subseteq R_{j}^{\mathcal{F}_{1} \vee \mathscr{F}_{2}}(A)$
(2) $\bar{R}_{j}^{j \mathcal{F}_{1} \vee \mathscr{F}_{2}}(A) \subseteq \bar{R}_{j}^{\mathcal{F}_{1}, \mathscr{F}_{2}}(A) \subseteq \bar{R}_{j}^{\mathscr{F}_{k}}(A) \subseteq \bar{R}_{j}(A)$

\section{Proof}

(1) $R(A) \subseteq R_{j}^{\mathscr{F}_{k}}(A) \subseteq R_{j}^{\mathscr{F}_{1}, \mathscr{F}_{2}}$ by Proposition 4. To prove, $R_{j_{f_{1}}, \mathscr{F}_{2}}^{j^{j}}(A) \subseteq R_{j}^{\mathcal{F}_{1} \vee \mathcal{F}_{2} j}(A)$. $R_{j}^{\mathcal{J}_{1}, \mathscr{F}_{2}}(A)=R_{j}^{\mathscr{F}_{1}}(A) \cup R_{j}^{\mathscr{F}_{2}}(A)$ and since $R_{j}^{\mathcal{F}_{k}}(A)=$ $\cup\left\{G \in \tau_{i}{ }^{\prime}: G \subseteq A\right\} \subseteq U$ $\left\{G \in \tau_{j}^{\mathcal{F}_{1} \mathcal{V} \dot{\mathcal{F}}_{2}}: G \subseteq A\right\}=R_{j}^{\mathscr{F}_{1} \vee \mathscr{F}_{2}}(A)$ (by Theorem 5), $R_{j}^{\mathscr{F}_{1}, \mathscr{F}_{2}}(A) \subseteq R_{j}^{\mathcal{F}_{1} \vee \mathscr{F}_{2}}(A)$.

(2) It is similar to (1).

Corollary 3. Let $\left(U, R, \xi_{j}\right)$ be a $j-N S, \mathscr{I}_{1} \vee \mathscr{I}_{2}$ an ideal on $U$, and $A \subseteq U$. Then, $\forall j \in\{r, l,\langle r\rangle,\langle l\rangle, i, u,\langle i\rangle,\langle u\rangle\}$ and $\forall k \in\{1,2\}$.
(1) $B_{j}^{\mathscr{F}_{1} \vee \mathscr{F}_{2}}(A) \subseteq B_{j}^{\mathscr{F}_{1}, \mathscr{F}_{2}}(A) \subseteq B_{j}^{\mathscr{F}_{k}}(A) \subseteq B_{j}(A)$
(2) $\sigma_{j}(A) \leq \sigma_{j}^{\mathscr{F}_{k}}(A) \leq \sigma_{j}^{\mathscr{F}_{1}, \mathscr{F}_{2}}(A) \leq \sigma_{j}^{\mathscr{F}_{1} \vee \mathscr{F}_{2}}(A)$

Corollary 4. Let $\left(U, R, \xi_{j}\right)$ be a $j-N S, \mathscr{I}_{1} \vee \mathscr{I}_{2}$ an ideal on $U$, and $A \subseteq U$. Then, $\forall j \in\{r, l,\langle r\rangle,\langle l\rangle, i, u,\langle i\rangle,\langle u\rangle\}$ and $\forall k \in\{1,2\}$.

(1) $A$ is j-exact $\Rightarrow A$ is $\mathscr{I}_{k}$-j-exact $\Rightarrow A$ is $\mathscr{I}_{1}, \mathscr{I}_{2}$-j-exact $\Rightarrow A$ is $\mathscr{I}_{1} \vee \mathscr{I}_{2}$-j-exact

(2) $A$ is $\mathscr{I}_{1} \vee \mathscr{I}_{2}$-j-rough $\Rightarrow A$ is $\mathscr{I}_{1}, \mathscr{I}_{2}$-j-rough $\Rightarrow A$ is

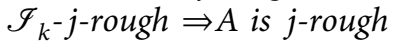

\section{Remark 7}

(1) The converse of parts of Theorem 7 and Corollaries 3 and 4 is not necessarily to be true as shown in Table 2 . This table is calculated by using Example 1 .

(2) Theorem 7 shows that the present method in Definitions 12 and 14 reduces the boundary region by increasing the lower approximations and decreasing the upper approximations with the comparison of Abd El-Monsef et al.'s Definition 6 [35] and Hosny's Definition 9 [28]. Moreover, Corollary 3 shows that the current accuracy in Definitions 12 and 14 is greater than the previous ones in Definitions 6 and 9 .
The following propositions and corollaries are studied the relationships among the $\mathscr{I}_{1} \vee \mathscr{I}_{2}$-j-lower, $\mathscr{I}_{1} \vee \mathscr{I}_{2}$-j-upper approximations, $\mathscr{I}_{1} \vee \mathscr{I}_{2}$-j-boundary regions, and $\mathscr{I}_{1} \vee \mathscr{I}_{2-j}$-accuracy.

Proposition 6. Let $\left(U, R, \xi_{j}\right)$ be a $j-N S, \mathscr{I}_{1} \vee \mathscr{I}_{2}$ an ideal on $U$, and $A \subseteq U$. Then, $\forall j \in\{r, l,\langle r\rangle,\langle l\rangle, i, u,\langle i\rangle,\langle u\rangle\}$. Then, the following statements are true in general:
(1) $R_{u}^{\mathscr{I}_{1} \vee \mathscr{F}_{2}}(A) \subseteq R_{r}^{\mathcal{F}_{1} \vee \mathscr{F}_{2}}(A) \subseteq R_{i}^{\mathcal{J}_{1} \vee \mathscr{F}_{2}}(A)$
(2) $R_{u}^{\mathscr{F}_{1} \vee \mathscr{F}_{2}}(A) \subseteq R_{l}^{\mathscr{F}_{1} \vee \mathscr{F}_{2}}(A) \subseteq R_{i}^{\mathscr{J}_{1} \vee \mathscr{F}_{2}}(A)$
(3) $R_{\langle u\rangle}^{\mathscr{F}_{1} \vee \mathscr{F}_{2}}(A) \subseteq R_{\langle r\rangle}^{\mathscr{F}_{1} \vee \mathscr{F}_{2}}(A) \subseteq R_{\langle i\rangle}^{\mathscr{F}}(A)$
(4) $R_{\langle u\rangle}^{\mathscr{F}_{1} \vee \mathscr{F}_{2}}(A) \subseteq R_{\langle l\rangle}^{\mathscr{F}_{1} \vee \mathscr{F}_{2}}(A) \subseteq R_{\langle i\rangle}^{\mathscr{F}_{1} \vee \mathscr{F}_{2}}(A)$

Proof. By using Proposition 2, the proof is obvious.

Proposition 7. Let $\left(U, R, \xi_{j}\right)$ be a $j-N S, \mathscr{I}_{1} \vee \mathscr{I}_{2}$ an ideal on $U$, and $A \subseteq U$. Then, $\forall j \in\{r, l,\langle r\rangle,\langle l\rangle, i, u,\langle i\rangle,\langle u\rangle\}$. Then, the following statements are true in general:
(1) $\bar{R}_{i}^{\mathscr{J}_{1} \vee \mathscr{F}_{2}}(A) \subseteq \bar{R}_{r}^{\mathscr{J}_{1} \vee \mathscr{F}_{2}}(A) \subseteq \bar{R}_{u}^{\mathscr{F}_{1} \vee \mathscr{F}_{2}}(A)$
(2) $\bar{R}_{i}^{\mathcal{F}_{1} \vee \mathscr{F}_{2}}(A) \subseteq \bar{R}_{l}^{\mathscr{I}}(A) \subseteq \bar{R}_{u}^{\mathscr{J}}(A)$
(3) $\bar{R}_{\langle i\rangle}^{\mathcal{F}_{1} \vee \mathscr{F}_{2}}(A) \subseteq \bar{R}_{\langle r\rangle}^{\mathcal{F}_{1} \vee \mathscr{F}_{2}}(A) \subseteq \bar{R}_{\langle u\rangle}^{\mathcal{F}_{1} \vee \mathscr{F}_{2}}(A)$
(4) $\bar{R}_{\langle i\rangle}^{\mathscr{J}_{1} \vee \mathscr{F}_{2}}(A) \subseteq \bar{R}_{\langle l\rangle}^{\mathscr{J}_{1} \vee \mathscr{F}_{2}}(A) \subseteq \bar{R}_{\langle u\rangle}^{\mathcal{I}_{1} \vee \mathscr{F}_{2}}(A)$

Proof. By using Proposition 2, the proof is obvious.

Corollary 5. Let $\left(U, R, \xi_{j}\right)$ be a $j-N S, \mathscr{I}_{1} \vee \mathscr{I}_{2}$ an ideal on $U$, and $A \subseteq U$. Then, $\forall j \in\{r, l,\langle r\rangle,\langle l\rangle, i, u,\langle i\rangle,\langle u\rangle\}$.
(1) $B_{i}^{\mathscr{G}_{1} \vee \mathscr{F}_{2}}(A) \subseteq B_{r}^{\mathcal{F}_{1} \vee \mathscr{F}_{2}}(A) \subseteq B_{u}^{\mathcal{F}_{1} \vee \mathscr{F}_{2}}(A)$
(2) $B_{i}^{\mathscr{F}_{1} \vee \mathscr{F}_{2}}(A) \subseteq B_{l}^{\mathcal{F}_{1} \vee \mathscr{F}_{2}}(A) \subseteq B_{u}^{\mathcal{F}_{1} \vee \mathscr{F}_{2}}(A)$
(3) $B_{\langle i\rangle}^{\mathscr{F}_{1} \vee \mathscr{F}_{2}}(A) \subseteq B_{\langle r\rangle}^{\mathcal{F}_{1} \vee \mathscr{F}_{2}}(A) \subseteq B_{\langle u\rangle}^{\mathcal{F}_{1} \vee \mathscr{F}_{2}}(A)$
(4) $B_{\langle i\rangle}^{\mathcal{F}_{1} \vee \mathscr{F}_{2}}(A) \subseteq B_{\langle l\rangle}^{\mathcal{F}_{1} \vee \mathscr{F}_{2}}(A) \subseteq B_{\langle u\rangle}^{\mathcal{F}_{1} \vee \mathscr{F}_{2}}(A)$

Corollary 6. Let $\left(U, R, \xi_{j}\right)$ be a $j-N S, \mathscr{I}_{1} \vee \mathscr{I}_{2}$ an ideal on $U$, and $A \subseteq U$. Then, $\forall j \in\{r, l,\langle r\rangle,\langle l\rangle, i, u,\langle i\rangle,\langle u\rangle\}$ :
(1) $\sigma_{u}^{\mathscr{F}_{1} \vee \mathscr{F}_{2}}(A) \leq \sigma_{r}^{\mathscr{F}_{1} \vee \mathscr{F}_{2}}(A) \leq \sigma_{i}^{\mathscr{F}_{1} \vee \mathscr{F}_{2}}(A)$
(2) $\sigma_{u}^{\mathscr{I}_{1} \vee \mathscr{F}_{2}}(A) \leq \sigma_{1}^{\mathscr{F}_{1} \vee \mathscr{F}_{2}}(A) \leq \sigma_{i}^{\mathscr{F}_{1} \vee \mathscr{F}_{2}}(A)$
(3) $\sigma_{\langle u\rangle}^{\mathscr{F}_{1} \vee \mathscr{F}_{2}}(A) \leq \sigma_{\langle r\rangle}^{\mathscr{F}_{1} \vee \mathscr{F}_{2}}(A) \leq \sigma_{\langle i\rangle}^{\mathscr{F}_{1} \vee \mathscr{F}_{2}}(A)$
(4) $\sigma_{\langle u\rangle}^{\mathscr{F}_{1} \vee \mathscr{F}_{2}}(A) \leq \sigma_{\langle l\rangle}^{\mathscr{F}_{1} \vee \mathscr{F}_{2}}(A) \leq \sigma_{\langle i\rangle}^{\mathscr{Y} \vee \mathscr{F}_{2}}(A)$

Remark 8. Let $\left(U, R, \xi_{j}\right)$ be a $j$-NS and $\mathscr{I}_{1} \vee \mathscr{I}_{2}$ an ideal on $U$. Then, the following should be noted that

(1) $\sigma_{r}^{\mathscr{J}_{1} \vee \mathscr{F}_{2}}(A)$ and $\sigma_{\langle r\rangle}^{\mathscr{F}_{1} \vee \mathscr{F}_{2}}(A)$ are not necessarily to be comparable

(2) $\sigma_{l}^{\mathscr{J}_{1} \vee \mathscr{F}_{2}}(A)$ and $\sigma_{\langle l\rangle}^{\mathscr{F}_{1} \vee \mathscr{F}_{2}}(A)$ are not necessarily to be comparable

(3) $\sigma_{i}^{\mathcal{F}_{1} \vee \mathscr{F}_{2}}(A)$ and $\sigma_{\langle i\rangle}^{\mathscr{F}_{1} \vee \mathscr{F}_{2}}(A)$ are not necessarily to be comparable

(4) $\sigma_{u}^{\mathscr{F}_{1} \vee \mathscr{F}_{2}}(A)$ and $\sigma_{\langle u\rangle}^{\mathscr{F}_{1} \vee \mathscr{F}_{2}}(A)$ are not necessarily to be comparable 


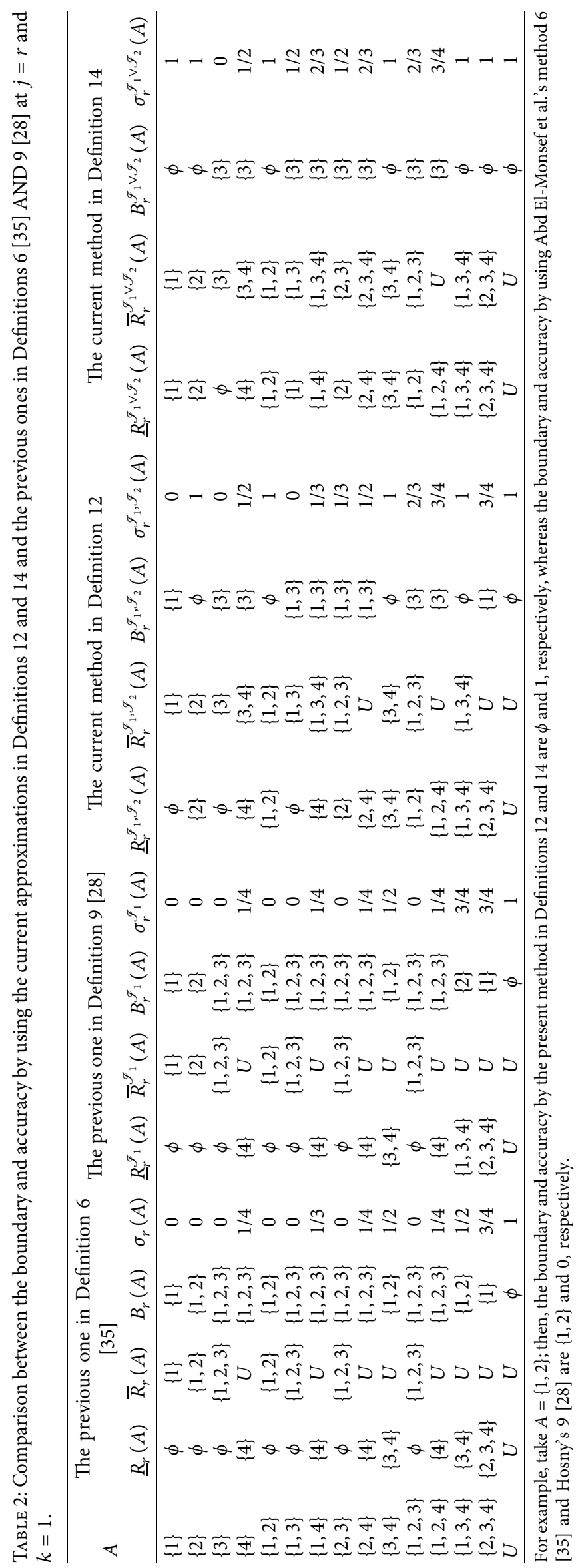


TABLe 3: Quantitative attributes of five amino acids.

\begin{tabular}{lccccccc}
\hline & $a_{1}$ & $a_{2}$ & $a_{3}$ & $a_{4}$ & $a_{5}$ & $a_{6}$ \\
\hline$\left\{x_{1}\right\}$ & -0.11 & -0.22 & 0.29 & 335 & 3.458 & -1.19 \\
$\left\{x_{2}\right\}$ & -0.51 & -0.64 & 0.76 & 311.6 & 3.243 & -1.43 \\
$\left\{x_{3}\right\}$ & 0 & 0 & 0 & 224.9 & 1.662 & 0.03 \\
$\left\{x_{4}\right\}$ & 0.15 & 0.13 & -0.25 & 337.2 & 3.856 & -1.06 \\
$\left\{x_{5}\right\}$ & 1.2 & 1.8 & -2.1 & 322.6 & 3.35 & 0.04 \\
\hline
\end{tabular}

TABLE 4: Right neighborhood of the seven relations.

\begin{tabular}{|c|c|c|c|c|c|c|c|}
\hline & $x_{i} R_{1}$ & $u_{i} R_{2}$ & $u_{i} R_{3}$ & $u_{i} R_{4}$ & $u_{i} R_{5}$ & $u_{i} R_{6}$ & $u_{i} R_{7}$ \\
\hline$\left\{x_{1}\right\}$ & $\left\{x_{1}, x_{3}, x_{4}, x_{5}\right\}$ & $U$ & $\left\{x_{1}, x_{2}, x_{3}, x_{4}\right\}$ & $\left\{x_{1}, x_{4}, x_{5}\right\}$ & $\left\{x_{1}, x_{2}, x_{4}, x_{5}\right\}$ & $U$ & $\left\{x_{1}, x_{2}, x_{4}, x_{5}\right\}$ \\
\hline$\left\{x_{2}\right\}$ & $U$ & $U$ & $\left\{x_{1}, x_{2}\right\}$ & $\left\{x_{1}, x_{2}, x_{4}, x_{5}\right\}$ & $\left\{x_{1}, x_{2}, x_{4}, x_{5}\right\}$ & $U$ & $\left\{x_{1}, x_{2}, x_{4}, x_{5}\right\}$ \\
\hline$\left\{x_{3}\right\}$ & $\left\{x_{1}, x_{3}, x_{4}, x_{5}\right\}$ & $\left\{x_{1}, x_{3}, x_{4}, x_{5}\right\}$ & $\left\{x_{1}, x_{2}, x_{3}, x_{4}\right\}$ & $U$ & $U$ & $\left\{x_{3}, x_{5}\right\}$ & $U$ \\
\hline$\left\{x_{4}\right\}$ & $\left\{\begin{array}{c}\left\{x_{1}, x_{3}, x_{4}, x_{5}\right\} \\
\left\{x_{-}\right\}\end{array}\right.$ & $\left\{\begin{array}{c}\left\{x_{1}, x_{3}, x_{4}, x_{5}\right\} \\
\left\{x_{-}\right\}\end{array}\right.$ & $\left\{x_{1}, x_{2}, x_{3}, x_{4}\right\}$ & $\left\{x_{1}, x_{4}, x_{5}\right\}$ & $\left\{x_{1}, x_{4}\right\}$ & $\left\{x_{1}, x_{3}, x_{4}, x_{5}\right\}$ & $\left\{x_{1}, x_{4}, x_{5}\right\}$ \\
\hline$\left\{x_{5}\right\}$ & $\left\{x_{5}\right\}$ & $\left\{x_{5}\right\}$ & U & $\left\{x_{1}, x_{2}, x_{4}, x_{5}\right\}$ & $\left\{x_{1}, x_{2}, x_{4}, x_{5}\right\}$ & $\left\{x_{3}, x_{5}\right\}$ & $\left\{x_{1}, x_{2}, x_{4}, x_{5}\right\}$ \\
\hline
\end{tabular}

Remark 9. Table 1 shows the following results:

(1) The comparison among the $\mathscr{I}_{1} \vee \mathscr{I}_{2}$-j-lower, $\mathscr{I}_{1} \vee \mathscr{I}_{2}$-j-upper $\mathscr{I}_{1} \vee \mathscr{I}_{2}$-j-boundary regions, and $\mathscr{I}_{1} \vee \mathscr{I}_{2}$-j-accuracy in Definition 14 by using Example 1 , for $j \in\{r, l, i, u\}$.

(2) There are different methods to approximate the sets by using $\tau_{j}^{\mathscr{J}_{1} \vee \mathscr{I}_{2}}, \forall j \in\{r, l, i, u\}$ in constructing the approximations of sets. The best one of these methods is given by using $\tau_{i}^{\mathscr{F}_{1} \vee \mathscr{I}_{2}}$ in constructing the approximations of sets since the boundary regions, in this case, are decreased (or canceled) by increasing the lower approximations and decreasing the upper approximations. Moreover, the $\mathscr{I}_{1} \vee \mathscr{I}_{2}$-i-accuracy is more accurate than the other types since $\sigma_{\sigma_{u} \vee \mathscr{F}_{2}}(A) \leq \sigma_{r}^{\mathscr{I}_{1} \vee \mathscr{F}_{2}}(A) \leq \sigma_{i_{1}}^{\mathscr{F}_{1} \vee \mathscr{F}_{2}}(A) \quad$ and $\sigma_{u}^{\mathscr{I} \vee} \vee \mathscr{F}_{2}(A) \leq \sigma_{l}^{\mathscr{F}_{1} \vee \mathscr{F}_{2}}(A) \leq \sigma_{i}^{\mathscr{F}_{1} \vee \mathscr{F}_{2}}(A)$.

It should be noted that the same as in Table 1 we can add an example to show the comparison among the $\mathscr{I}_{1} \vee \mathscr{I}_{2}$-j-lower, $\quad \mathscr{I}_{1} \vee \mathscr{I}_{2}$-j-upper approximations, $\mathscr{I}_{1} \vee \mathscr{I}_{2}$-j-boundary regions, and $\mathscr{I}_{1} \vee \mathscr{I}_{2}$-j-accuracy in Definition 14, for $j \in\{\langle r\rangle,\langle l\rangle,\langle i\rangle,\langle u\rangle\}$, and to illustrate that there are different methods to approximate the sets by using $\tau_{j}^{\mathscr{I}_{1} \vee \mathscr{F}_{2}}, \forall j \in\{\langle r\rangle,\langle l\rangle,\langle i\rangle,\langle u\rangle\}$ in constructing the approximations of sets. The best one of these methods is given by using $\tau_{\langle i\rangle}^{\mathscr{I}_{1} \vee \mathscr{I}_{2}}$ in constructing the approximations of sets since the boundary regions in this case are decreased (or canceled) by increasing the lower approximations and decreasing the upper approximations. Moreover, the

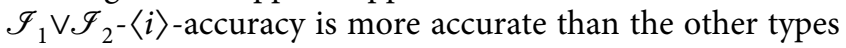
since $\quad \sigma_{\langle u\rangle}^{\mathscr{I}_{1} \vee \mathscr{F}_{2}}(A) \leq \sigma_{\langle r\rangle}^{\mathscr{I}_{1} \vee \mathscr{F}_{2}}(A) \leq \sigma_{\langle i\rangle}^{\mathscr{I}_{1} \vee \mathscr{F}_{2}}(A) \quad$ and $\sigma_{\langle u\rangle}^{\mathscr{F}_{1} \vee \mathscr{F}_{2}}(A) \leq \sigma_{\langle l\rangle}^{\mathcal{F}_{1} \vee \mathscr{F}_{2}}(A) \leq \sigma_{\langle i\rangle}^{\mathcal{F}_{1} \vee \mathscr{I}_{2}}(A)$

Remark 10. It should be noted that the relationships among the $\mathscr{I}_{1}, \mathscr{I}_{2}$-j-lower, $\mathscr{I}_{1}, \mathscr{I}_{2}$-j-upper approximations, $\mathscr{I}_{1}, \mathscr{I}_{2}$-j-boundary regions, and
$\mathscr{I}_{1}, \mathscr{I}_{2}$ - $j$-accuracy are the same as in Propositions 6 and 7 and Corollaries 5 and 6.

\section{Application}

Example 2. Let $U=\left\{x_{1}, x_{2}, x_{3}, x_{4}, x_{5}\right\}$ be five amino acids (for short, AAs). The AAs are described in terms of seven attributes: $a_{1}=$ PIE, $a_{2}=$ PIF (two measures of the side chain lipophilicity), $a_{3}=\mathrm{DGR}=\Delta G$ of transfer from the protein interior to water, $a_{4}=\mathrm{SAC}=$ surface area, $a_{5}=\mathrm{MR}=$ molecular refractivity, $a_{6}=\mathrm{LAM}=$ the side chain polarity, and $a_{7}=\mathrm{Vol}=$ molecular volume [36,37]. Table 3 shows all quantitative attributes of five AAs.

We consider the following seven relations on $U$ defined as follows: $R_{i}=\left\{\left(x_{i}, x_{j}\right): x_{i}\left(a_{k}\right)-\quad x_{j}\left(a_{k}\right)<\left(\sigma_{k} / 2\right)\right.$, $i, j=1,2, \ldots, 5, k=1,2, \ldots, 7\}$ where $\sigma_{k}$ represents the standard deviation of the quantitative attributes $a_{k}, k=1,2, \ldots, 7$. The right neighborhoods for all elements of $U=\left\{x_{1}, x_{2}, x_{3}, x_{4}, x_{5}\right\}$ with respect to the relations $R_{k}, k=$ $1,2, \ldots, 7$ are shown in Table 4 .

Therefore, we find the intersection of all right neighborhoods of all element $k=1,2, \ldots, 7$ as follows: $x_{1} R=$ $\cap_{k=1}^{7}\left(x_{1} R_{k}\right)=\left\{x_{1}, x_{4}\right\}, x_{2} R=\cap_{k=1}^{7}\left(x_{2} R_{k}\right)=\left\{x_{1}, x_{2}\right\}, x_{3} R=$ $\cap_{k=1}^{7}\left(x_{3} R_{k}\right)=\left\{x_{3}\right\}, x_{4} R=\cap_{k=1}^{7}\left(x_{4} R_{k}\right)=\left\{x_{1}, x_{4}\right\}, x_{5} R=$ $\cap_{k=1}^{7}\left(x_{5} R_{k}\right)=\left\{x_{5}\right\}$. Then, $R=\Delta \cup\left\{\left(x_{1}, x_{4}\right),\left(x_{2}, x_{1}\right)\right.$, $\left.\left(x_{4}, x_{1}\right)\right\}$, where $\Delta$ is the identity relation and equal to $\left\{\left(x_{1}, x_{1}\right),\left(x_{2}, x_{2}\right),\left(x_{3}, x_{3}\right),\left(x_{4}, x_{4}\right),\left(x_{5}, x_{5}\right)\right\}$. Let $\mathscr{I}_{1}=\{\phi$, $\left.\left\{x_{4}\right\}\right\}, \mathscr{I}_{2}=\left\{\phi,\left\{x_{1}\right\}\right\}$, and $\mathscr{I}_{1} \vee \mathscr{I}_{2}=\left\{\phi,\left\{x_{1}\right\},\left\{x_{4}\right\},\left\{x_{1}, x_{4}\right\}\right\}$. Then, we calculate the lower, upper approximations, boundary region, and accuracy by using Abd El-Monsef et al.'s Definition 6 [35] and Hosny's Definition 9 [28] and the current method in Definitions 12 and 14 as shown in Table 5. Hence, for any concept $A \subseteq U$ (collection of amino acid), this concept is determined by lower and upper approximations which define its boundary. The accuracy increases by the decrease of the boundary region. Clearly, the accuracy measure by using the current approximations in Definitions 12 and 14 is greater than 


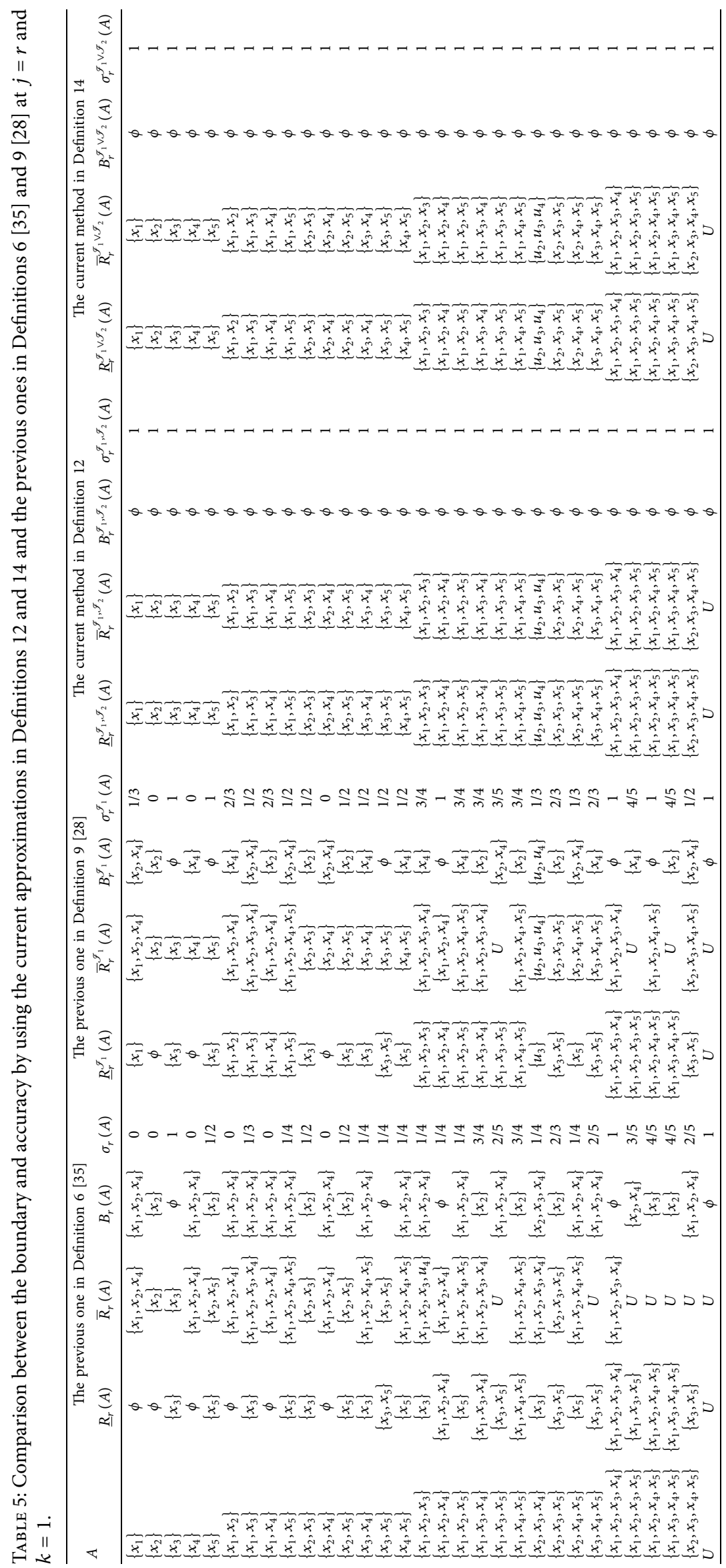


the accuracy measure by using Abd El-Monsef et al.'s Definition 6 [35] and Hosny's Definition 9 [28].

\section{Conclusions}

The rough theory is a new mathematical tool to deal with vagueness and imperfect knowledge by using the concept of the lower and upper approximations. If the lower and upper approximations of the set are equal to each other, then it is called a crisp (exact) set; otherwise, it is known as a rough (inexact) set. Therefore, the boundary region is defined as the difference between the upper and lower approximations, and then the accuracy of the set or ambiguous depending on the boundary region is empty or not respectively. A nonempty boundary region of a set means that our knowledge about the set is not sufficient to define the set precisely. The main aim of the rough set is to reduce the boundary region by increasing the lower approximation and decrease the upper approximation. An interesting and natural research topic in the rough set theory is to study the rough set theory via topology. The topology induced by binary relations on the universes and bi-ideals was used to generalize the basic rough set concepts. In this paper, different new types of rough set based on bi-ideals were defined to reduce the boundary region and increase the accuracy measure which is the main aim of rough set theory. The concepts of lower and upper approximations based on bi-ideals were presented for these types. Several properties and examples were provided. Additionally, some important properties and results of these approximations were instituted. The relationships between the present approximations and the previous approximations were established and shown to be more general. We conclude that the suggested topological operations and structures open the way for more topological applications in rough context. We can say that the improvements in abstract topology results help in some way in the modifications of rough sets theory and consequently in its real-life applications. It should be noted that any one can extend the current method similarly by using $n$-ideals. In the real life, this comparison is represented if we need to make a decision about a manuscript and send it to two reviewers $\mathscr{I}_{1}, \mathscr{I}_{2}$. Then, we have two cases:

(1) Take two reports separately from the referees and use one of the mathematical methods to find the final decision

(2) Take a combined report from the referees together

It is clear that the second case is better than the first one such as the approximations of the present study.

\section{Data Availability}

The data of the applied example in the chemistry field are taken from $[36,37]$. All this information is included in the manuscript.

\section{Conflicts of Interest}

The author declares no conflicts of interest.

\section{Acknowledgments}

The author extends her appreciation to the deanship of scientific research at King Khalid University for funding this work through a research group program under Grant no. R.G.P. 1/15/42.

\section{References}

[1] Z. Pawlak, "Rough sets," International Journal of Computer \& Information Sciences, vol. 11, no. 5, pp. 341-356, 1982.

[2] Z. Pawlak, "Rough concept analysis," Bulletin of the Polish Academy of Sciences-Mathematics, vol. 33, pp. 9-10, 1985.

[3] J. Järinen, "Approximations and rough sets based on tolerances," in Lecture Notes in Artificial Intelligence 2475(RSCTC 2002), W. Ziarko and Y. Yao, Eds., pp. 123-130, Springer, Berlin, Germany, 2002.

[4] M. Kondo, "On the structure of generalized rough sets," Information Sciences, vol. 176, pp. 589-600, 2005.

[5] M. Kryszkiewicz, "Rough set approach to incomplete information systems," Information Sciences, vol. 112, no. 1-4, pp. 39-49, 1998.

[6] J. Nieminen, "Rough tolerance equality and tolerance black boxes," Fundamenta Informaticae, vol. 11, no. 3, pp. 289-296, 1988.

[7] A. Skowron and J. Stepaniuk, "Tolerance approximation spaces," Fundamenta Informaticae, vol. 27, no. 2-3, pp. 245-253, 1996.

[8] R. Slowinski and J. Stefanowski, "Rough-set reasoning about uncertain data," Fundamenta Informaticae, vol. 27, pp. 229243, 1996.

[9] A. Wiweger, "On topological rough sets," Bulletin of the Polish Academy of Sciences-Mathematics, vol. 37, pp. 89-93, 1989.

[10] H. M. Abu-Donia and A. S. Salama, "Generalization of Pawlak's rough approximation spaces by using $\delta \beta$-open sets," International Journal of Approximate Reasoning, vol. 53, no. 7, pp. 1094-1105, 2012.

[11] J. Dai, S. Gao, and G. Zheng, "Generalized rough set models determined by multiple neighborhoods generated from a similarity relation," Soft Computing, vol. 13, pp. 2081-2094, 2013.

[12] E. F. Lashin, A. M. Kozae, A. A. Abo Khadra, and T. Medhat, "Rough set theory for topological spaces," International Journal of Approximate Reasoning, vol. 40, no. 1-2, pp. 35-43, 2005.

[13] X. Li and S. Liu, "Matroidal approaches to rough sets via closure operators," International Journal of Approximate Reasoning, vol. 53, no. 4, pp. 513-527, 2012.

[14] Z. Li, T. Xie, and Q. Li, "Topological structure of generalized rough sets," Computers \& Mathematics with Applications, vol. 63, no. 6, pp. 1066-1071, 2012.

[15] T. Y. Lin, Q. Liu, and Y. Y. Yao, Logic Systems for Approximate Reasoning: Via Rough Sets and Topology, Methodologies for Intelligent Systems, Springer-Verlag, Berlin, Germany, 1994.

[16] Z. Pei, D. Pei, and L. Zheng, "Topology vs generalized rough sets," International Journal of Approximate Reasoning, vol. 52, no. 2, pp. 231-239, 2011.

[17] K. Qin and Z. Pei, "On the topological properties of fuzzy rough sets," Fuzzy Sets and Systems, vol. 151, no. 3, pp. 601-613, 2005.

[18] E. A. Rady, A. M. Kozae, and M. M. E. Abd El-Monsef, "Generalized rough sets," Chaos, Solitons \& Fractals, vol. 21, no. 1, pp. 49-53, 2004. 
[19] A. S. Salama, "Some topological properties of rough sets with tools for data mining," International Journal of Computer Science, vol. 8, pp. 588-595, 2011.

[20] A. Skowron, "On topology in information system," Bulletin of the Polish Academy of Sciences-Mathematics, vol. 36, pp. 477-480, 1988.

[21] W. Z. Wu, "A study on relationship between fuzzy rough approximation operators and fuzzy topological spaces," Fuzzy Systems and Knowledge Discovery, vol. 3613, pp. 167-174, 2005.

[22] Q. Wu, T. Wang, Y. Huang, and J. Li, “Topology theory on rough sets," IEEE Transactions on Systems, Man, and Cybernetics, Part B (Cybernetics), vol. 38, no. 1, pp. 68-77, 2008.

[23] W. Zhu, "Topological approaches to covering rough sets," Information Sciences, vol. 177, no. 6, pp. 1499-1508, 2007.

[24] R. Vaidynathaswamy, "The localization theory in set topology," Proceedings of the Indiana Academy of Science, vol. 20, pp. 515-561, 1945.

[25] D. Jankovic and T. R. Hamlet, "New topologies from old via ideals," The American Mathematical Monthly, vol. 97, no. 4, pp. 295-310, 1990.

[26] T. M. Al-shami, H. Işik, A. S. Nawar, and R. A. Hosny, "Some topological approaches for generalized rough sets via ideals," Mathematical Problems in Engineering, vol. 2021, Article ID 5642982, 11 pages, 2021.

[27] M. Hosny, "Topological approach for rough sets by using $J$ nearly concepts via ideals," Filomat, vol. 34, no. 2, pp. 273-286, 2020.

[28] M. Hosny, "Idealization of $j$-approximation spaces," Filomat, vol. 34, no. 2, pp. 287-301, 2020.

[29] R. A. Hosny, B. A. Asaad, A. A. Azzam, and T. M. Al-Shami, "Various topologies generated from $E_{j}$-neighbourhoods via ideals," Complexity, vol. 2021, Article ID 4149368, 11 pages, 2021.

[30] M. Hosny and M. Raafat, "On generalization of rough multiset via multiset ideals," Journal of Intelligent and Fuzzy Systems, vol. 33, no. 2, pp. 1249-1261, 2017.

[31] A. Kandil, S. A. El-Sheikh, M. Hosny, and M. Raafat, "Bi-ideal approximation spaces and their applications," Soft Computing, vol. 24, pp. 12989-13001, 2020.

[32] A. M. Kozae, "On topology expansions by ideals and applications," Chaos, Solitons \& Fractals, vol. 13, no. 1, pp. 55-60, 2002.

[33] O. A. E. Tantawy and H. I. Mustafa, "On rough approximations via ideal," Information Sciences, vol. 251, pp. 114-125, 2013.

[34] M. E. Abd El-Monsef, A. S. Salama, and O. A. Embaby, "Granular computing using mixed neighborhood systems," Journal of International Journal of Mathematics and Computer Science, vol. 20, pp. 233-243, 2009.

[35] M. E. Abd El-Monsef, O. A. Embaby, and M. K. E. Bably, "Comparison between rough set approximations based on different topologies," International Journal of Granular Computing, Rough Sets and Intelligent Systems, vol. 3, no. 4, pp. 292-305, 2014.

[36] N. El Tayar, R. S. Tsai, P. A. Carrupt, and B. Testa, “Octan-1ol-water partition coefficients of zwitterionic $\alpha$-amino acids. Determination by centrifugal partition chromatography and factorization into steric/hydrophobic and polar components," Journal of the Chemical Society, Perkin Transactions, vol. 2, no. 1, pp. 79-84, 1992.

[37] B. Walczak and D. L. Massart, "Rough sets theory," Chemometrics and Intelligent Laboratory Systems, vol. 47, no. 1, pp. 1-16, 1999. 